Review

\title{
An Updated Meta-Analysis of Remote Blood Pressure Monitoring in Urban-Dwelling Patients with Hypertension
}

\author{
Sang-Hyun Park ${ }^{1,+} \oplus$, Jong-Ho Shin ${ }^{1,+}$, Joowoong Park ${ }^{2}$ and Woo-Seok Choi ${ }^{3,4, *(1)}$ \\ 1 Department of Internal Medicine, Daejeon Eulji Medical Center, Eulji University School of Medicine, \\ Daejeon 35233, Korea; psh@eulji.ac.kr (S.-H.P.); redsea98@eulji.ac.kr (J.-H.S.) \\ 2 Research Strategy Division, Korea Aerospace Research Institute (KARI), Daejeon 34133, Korea; \\ park@kari.re.kr \\ 3 Moon Soul Graduate School of Future Strategy, Korea Advanced Institute of Science and Technology (KAIST), \\ Daejeon 34141, Korea \\ 4 Keyu Internal Medicine Clinic, Daejeon 35250, Korea \\ * Correspondence: keyuim@kaist.ac.kr; Tel.: +82-42-483-7554; Fax: +82-42-485-7554 \\ + These authors contributed equally to this work.
}

\section{check for}

updates

Citation: Park, S.-H.; Shin, J.-H. Park, J.; Choi, W.-S. An Updated Meta-Analysis of Remote Blood Pressure Monitoring in

\section{Urban-Dwelling Patients with} Hypertension. Int. J. Environ. Res. Public Health 2021, 18, 10583. https:// doi.org/10.3390/ijerph182010583

\section{Academic Editors:}

Irene Torres-Sanchez and Marie Carmen Valenza

Received: 23 September 2021

Accepted: 5 October 2021

Published: 9 October 2021

Publisher's Note: MDPI stays neutral with regard to jurisdictional claims in published maps and institutional affiliations.

Copyright: (c) 2021 by the authors. Licensee MDPI, Basel, Switzerland. This article is an open access article distributed under the terms and conditions of the Creative Commons Attribution (CC BY) license (https:// creativecommons.org/licenses/by/ $4.0 /)$.

\begin{abstract}
Following the coronavirus disease-2019 pandemic, this study aimed to evaluate the overall effects of remote blood pressure monitoring (RBPM) for urban-dwelling patients with hypertension and high accessibility to healthcare and provide updated quantitative summary data. Of 2721 database-searched articles from RBPM's inception to November 2020, 32 high-quality studies (48 comparisons) were selected as primary data for synthesis. A meta-analysis was undertaken using a random effects model. Primary outcomes were changes in office systolic blood pressure (SBP) and diastolic blood pressure (DBP) following RBPM. The secondary outcome was the BP control rate. Compared with a usual care group, there was a decrease in SBP and DBP in the RBPM group (standardized mean difference 0.507 (95\% confidence interval [CI] 0.339-0.675, $p<0.001$; weighted mean difference [WMD] $4.464 \mathrm{mmHg}, p<0.001$ ) and 0.315 (CI 0.209-0.422, $p<0.001$; WMD $2.075 \mathrm{mmHg}$, $p<0.001)$, respectively). The RBPM group had a higher BP control rate based on a relative ratio (RR) of $1.226(1.107-1.358, p<0.001)$. RBPM effects increased with increases in city size and frequent monitoring, with decreases in intervention duration, and in cities without medically underserved areas. RBPM is effective in reducing BP and in achieving target BP levels for urban-dwelling patients with hypertension.
\end{abstract}

Keywords: blood pressure; remote monitoring; hypertension; telemedicine; urban

\section{Introduction}

Hypertension is widely recognized as the most important risk factor for cardiovascular disease (CVD), which is a major cause of total mortality [1]. A $2 \mathrm{mmHg}$ fall in systolic blood pressure (SBP) has been reported to reduce the incidence of ischemic CVD and stroke by $7 \%$ [2]. However, even in advanced countries, target blood pressure (BP) is achieved in $<50 \%$ of patients with hypertension [3,4]. The 2017 American College of Cardiology/American Heart Association (ACC/AHA) and 2018 European Society of Cardiology /European Society of Hypertension (ESC/ESH) treatment recommendations state that BP must be controlled to stricter levels $[5,6]$.

Remote BP monitoring (RBPM) has been recommended for hypertension diagnosis and treatment $[5,6]$, as it has been reported to predict CVD morbidity and mortality with higher accuracy than office BP monitoring [7]. As a method of telemedicine, RBPM is known to be an effective tool to enhance drug adherence and BP control in patients with hypertension [8-12]. RBPM has been suggested as a potential solution to overcome the geographical limitations of healthcare services [13], with significant effects shown in randomized controlled trials (RCTs) and meta-analysis studies [10,14-16]. The 2017 
ACC / AHA guidelines also recommended RBPM for hypertension diagnosis and control, and for enhancing patients' drug adherence [6].

According to the United Nations, approximately $68 \%$ of the human population is predicted to dwell in urban settings by 2050 [17]. Urbanization is a rapidly growing 21 st century trend, with significant effects on human health. However, despite increased interest in new health technologies, several studies have reported that remote monitoring has limited application in urban settings where high-quality face-to-face care is possible and healthcare accessibility is high $[18,19]$. Moreover, there is no comprehensive evidence concerning the effect of RBPM in improving clinical outcomes of urban-dwelling patients with hypertension or whether RBPM can become a standard treatment for hypertension management.

In a previous meta-analysis of RCTs using the Jovell/Navarro-Rubio classification system to determine the strength of evidence, RBPM showed statistically significant reductions in SBP (3.48 $\mathrm{mmHg}$ ) and diastolic BP (DBP, $1.64 \mathrm{mmHg}$ ) compared with usual care (UC) after an average of 7.6 months for patients dwelling in an urban setting. In terms of CVD prevention, however, RBPM induced $<0.5 \%$ of CVD prevention in low-risk patients with hypertension. Therefore, some studies have concluded that RBPM is of little practical significance to policy-makers [20,21]. The coronavirus disease-2019 (COVID-19) pandemic resulted in a steeply increased demand for telemedicine, even in urban settings, for those otherwise having adequate availability and accessibility to healthcare services. More generally, characteristically dense populations in cities have resulted in the rapid spread of infectious diseases, leading to the expansion of infrastructure for non-face-to-face care in line with a rapid increase in the use of the internet and mobile devices.

Considering the global rate of BP control, according to 2017 ACC / AHA guidelines for hypertension diagnosis and control, which is the latest strict guideline for hypertension diagnosis and control, the proportion of patients achieving the target BP is predicted to decrease further. The use of remote medical care services suddenly increased during the COVID-19 pandemic [14,22,23], and its use needs to be verified based on the integration of previous findings, given that hypertension is a chronic disease requiring long-term management for CVD prevention and for efficient healthcare policies to be implemented in urban settings. Therefore, relevant studies need to be extended through an updated compilation of BP data. The objective of our study is to evaluate whether RBPM could be utilized as an alternative to standard treatment for urban-dwelling patients with hypertension during the COVID-19 pandemic. Thus, this study aimed to determine the relative effects of RBPM compared with UC based on outcomes including SBP, DBP, and BP control rates. Intervention duration, city size, setting, frequency of remote transmission of $\mathrm{BP}$ data, and the presence of medically underserved areas (MUAs) in the city were analyzed as secondary factors to evaluate the effects of RBPM. We hypothesized that the effects of RBPM were equivalent to those of UC. To test this hypothesis, relevant, up-to-date RCTs were systematically reviewed and transparent and reliable quantitative data synthesis was performed.

\section{Materials and Methods}

\subsection{Searching for Eligible Studies}

This study followed the Preferred Reporting Items for Systematic Reviews and MetaAnalyses (PRISMA) guidelines of the Cochrane Collaboration and a checklist was provided [Supplementary Materials] [24]. To identify eligible studies, two investigators (SHP and JHS) independently searched the following electronic databases: PubMed, EBSCOhost, Embase, and the Cochrane Library, from RBPM's inception to November 30, 2020. Free terms were used, including and related to urban, hypertension, and remote monitoring, along with medical subject heading $(\mathrm{MeSH})$ terms. Truncation and phrasing methods were applied to derive a structured search formula [20] (Appendix A). The formula was first applied to the Cochrane Library and then converted to suit each database for the subsequent search. Articles written in English were retrieved. To include as many relevant articles 
as possible, all systematic reviews and meta-analyses related to the search themes were collected from each database and Google Scholar, and their reference lists were reviewed. To identify gray literature, relevant websites were used, and all studies including those in which the city area was not clearly defined were identified through a manual search.

\subsection{Inclusion and Exclusion Criteria}

All included studies were blinded RCT studies with random and uniform allocation of patients with hypertension into an RBPM group and a traditional face-to-face UC group. Articles reporting pre- and post-intervention data were targeted, with participants satisfying the following criteria: (1) patients with hypertension under management through regular visits to an urban medical institution; (2) patients able to measure their own BP at home; (3) patients able to transmit their BP data to the physician via post, phone, Bluetooth device, mobile phone, web, or computer (wired or wireless); (4) adults aged $\geq 18$ years; (5) BP measurement through ambulatory monitoring; and (6) various transmission methods from real-time or a stored and forward method to an automatic or manual method. Exclusion criteria comprised the following: (1) sudden BP changes due to an acute CVD or cerebrovascular accident (CVA); (2) patients undergoing hemodialysis due to acute or chronic renal disease; (3) female patients before and after pregnancy; (4) cases not reported for urban areas or cases for urban and rural areas reported together; (5) cases from unclear target areas; (6) cases where monitoring was aided by medical staff at a nursing management unit or care facility; and (7) cluster trials or cross-over studies.

\subsection{Study Selection}

The citations retrieved from each database were exported to EndNote X8.2, and two investigators (SHP and JHS) independently eliminated those not satisfying the criteria to confirm the reliability of identification. First, the title and abstract were screened, and for studies satisfying the criteria, full texts were obtained and scrutinized. Primary studies were selected independently, and their reference lists were reviewed. Final articles for data synthesis were determined after discussion with the senior author (WSC).

\subsection{Data Extraction and Coding}

For the selected studies, data extraction was performed independently by two investigators (JHS and WSC), and relevant values were coded in an electronic sheet. The extracted data included demographic and pre- and post-intervention SBP and DBP data. $\mathrm{BP}$ data were mostly obtained using an automated device and, in the case of ambulatory BP monitoring (ABPM), the mean of each group was calculated and coded. If an article did not report BP values or standard deviations (SDs), preventing calculations with a $95 \%$ confidence interval, the values were first checked on the trial registries website and, in cases where the required information could not be obtained, an attempt was made to contact the author of the article $[25,26]$. Articles that satisfied the inclusion criteria but did not report the main BP data were excluded from the final data synthesis. For some studies with missing SDs, data imputation was performed using a simple method [27,28]. The mean of all other studies, excluding those with missing data, was obtained. Regarding the rate of $\mathrm{BP}$ control, the number of patients satisfying the level of normal BP, determined during the final follow-up period of comparison in each study, was calculated and compared between the two groups. If a single primary study included several different follow-up periods for comparison [26-35]; applied a different, additional intervention [25,34]; or had multiple varying sample sizes and thus reported varying results, each result was included in the analysis as an independent study. Disagreements between investigators were resolved through consultation with the senior author (WSC).

\subsection{Quality Assessment and Publication Bias}

The quality assessment of the primary studies included evaluating the risk of bias (RoB) and was performed independently by two investigators (SHP and JP). Using the 
Review Manager program (RevMan, version 5.3.5, Copenhagen, Denmark) software from the Cochrane Collaboration, the evaluation was performed according to the Cochrane Handbook for Systematic Reviews of Interventions guidelines [24,36]. Disagreements were resolved through discussion among investigators. To identify publication bias, Egger's regression, classic fail-safe N, Duval and Tweedie's trim-and-fill method, and funnel plots were used.

\subsection{Statistical Analysis}

To ensure the reliability of the analysis, coded data were analyzed by two investigators (SHP \& JHS) using Comprehensive Meta-Analysis version 2 (CMA, Biostat, Englewood, NJ, USA) software. For primary outcomes, continuous variables comprised the weighted mean difference (WMD) and the standardized mean difference (SMD) obtained from the mean SBP and DBP values measured at baseline and during follow-up in the office. Despite divided opinions regarding the use of continuous variables, SMD has shown a trend of higher statistically significant generalizability and percentage agreement than the WMD in a random effects model (REM) and a fixed effects model (FEM) [37,38]. Therefore, SMD was used in this study to report the results of the data synthesis for continuous variables. Considering the generalizability of each result, the WMD was additionally estimated for comparing the subgroup results [38]. Based on Cohen's general rule of thumb, the effect size was set as follows: SMD 0.2 (small effect); SMD 0.5 (medium effect), and SMD 0.8 (large effect) [39]. Accordingly, when the SMD was $\geq 0.5$, we considered the effect size to be significant in this study. The rate of BP control was a dichotomous variable, for which $\mathrm{BP}$ normalization data were extracted from each study, and effect size based on relative risk (RR) was used. A 95\% confidence interval (CI) was used for all data. To analyze the inter-rater difference, a $\chi^{2}$ test was used and the level of significance was set to $p<0.10$. The model of analysis was applied after assessing the enrolled population of each study and the heterogeneity among research centers. Between-study heterogeneity was presented using Tau-squared $\left(\tau^{2}\right)$ and I-squared $\left(\mathrm{I}^{2}\right)$ indices, and the adequacy of results was determined based on Cohen's general rule of thumb [40]. Therefore, in this study, $30 \leq \mathrm{I}^{2} \leq 60$ indicated moderate heterogeneity and $50 \leq \mathrm{I}^{2} \leq 90$ indicated substantial heterogeneity [39]. To assess the quality of each trial and the consequent impact on the overall effect size, sensitivity was tested using the "one study removed" method (Appendix B, Figure A1). A cumulative analysis was run for a total of 48 comparisons, and the range of summary effect sizes at each step according to temporal progression was determined. $p$-values and the presence of outliers affecting the overall effect size were also determined (Appendix C, Figure A2). An additional sensitivity test was performed to determine differences between the data before and after imputing the missing values.

\section{Results}

\subsection{Study Characteristics}

Through an initial search of available databases, reference to trial registries, and a manual search of reference lists, a total of 2721 citations were retrieved (Figure 1). Of these, 992 duplicates were removed, leaving 1729 citations to be identified. Next, titles and abstracts for each identified citation were screened, and 1217 irrelevant citations were excluded. For the remaining 512 articles, the full text was obtained and scrutinized, and studies without available data $(n=206)$, studies not performed in an urban area, studies either reporting combined results of urban and rural areas or not reporting the area $(n=192)$, studies conducted on patients with CVD or CVA that may induce a sudden change in BP, studies conducted on patients undergoing hemodialysis or including patients with chronic renal disease, and studies involving female patients before or after pregnancy $(n=46)$ or patients aged $<18$ years $(n=21)$ were excluded. In total, 32 independent studies (48 comparisons) satisfying the inclusion criteria were used in the final data synthesis (Table 1). 


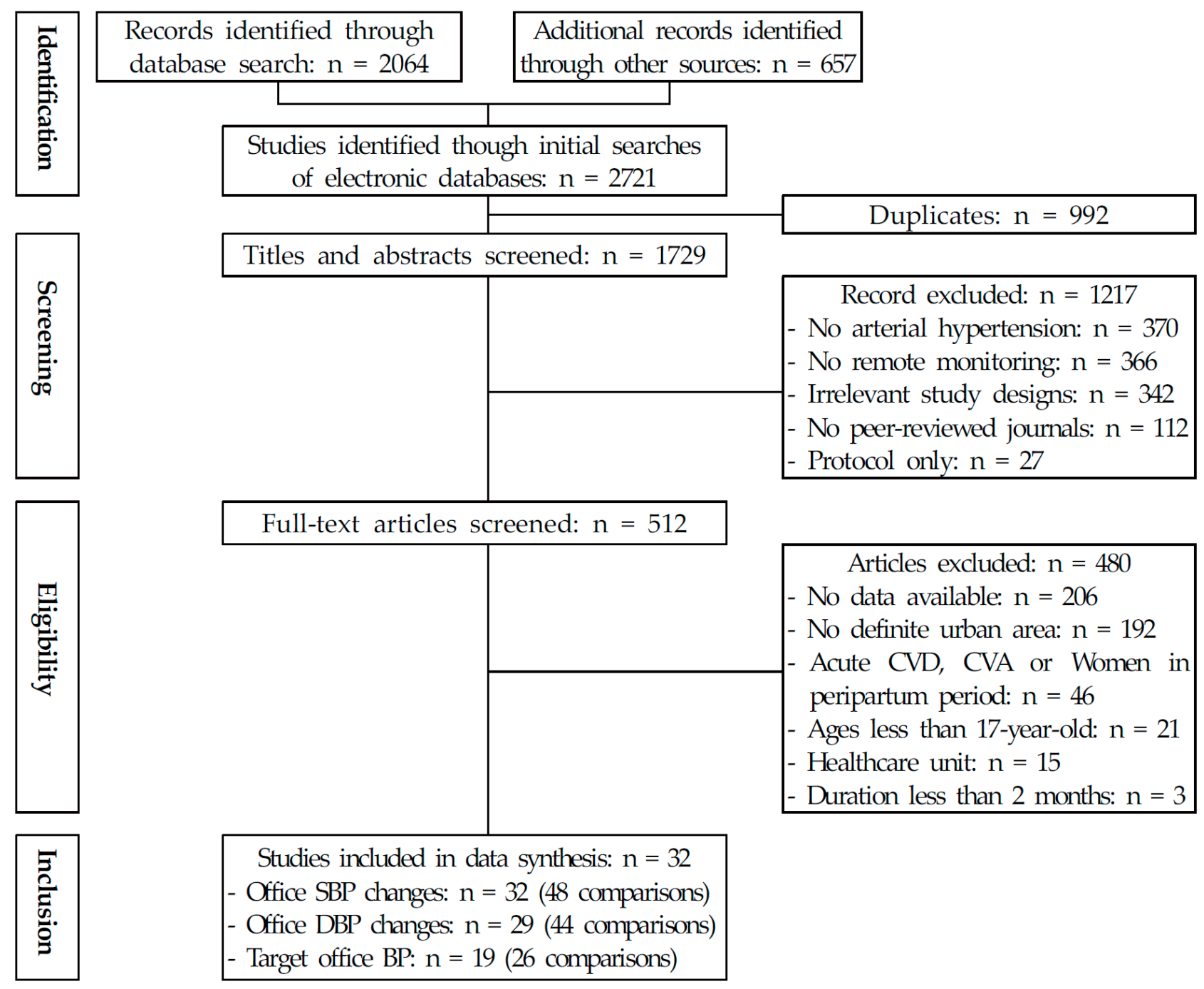

Figure 1. PRISMA flow of study. Abbreviations: BP, blood pressure; CVD, cardiovascular disease; CVA, cerebro-vascular accident; DBP, diastolic blood pressure; SBP, systolic blood pressure.

For the primary studies included in the meta-analysis in this study, the duration of RBPM was 2-18 months (mean, 7.37 months), and the number of participants in the UC and RBPM groups was 5666 and 5729, respectively. The mean age of participants in the UC and RBPM groups was 52.63 and 52.17 years, respectively. No significant intergroup differences were found in terms of sex and baseline BP. No differences in ethnicity were observed. Fourteen studies were conducted in primary medical institutions, 12 in community healthcare centers, and 22 in hospitals or higher-level institutions. The completion dates were in or prior to the year 2000 for two studies [41,42], between 2001 and 2010 for 14 studies [25,29,43-50], and between 2011 and 2020 for 32 studies. Seven studies had used mean values for ABPM [47,48,50-54]. 
Table 1. Characteristics of individual primary studies.

\begin{tabular}{|c|c|c|c|c|c|c|c|c|c|c|c|c|c|}
\hline \multirow{2}{*}{ Study } & \multirow{2}{*}{$\begin{array}{c}\text { Included } \\
\text { Participants }\end{array}$} & \multicolumn{2}{|c|}{$\begin{array}{l}\text { Participants } \\
\text { Number }\end{array}$} & \multicolumn{3}{|c|}{$\begin{array}{c}\text { Participants' Age } \\
\text { Interval (Years) }\end{array}$} & \multirow{2}{*}{$\begin{array}{l}\text { Duration } \\
\text { (Months) }\end{array}$} & \multirow{2}{*}{$\begin{array}{c}\text { City } \\
\text { Name } \\
\text { (Country) }\end{array}$} & \multirow{2}{*}{$\begin{array}{l}\text { Population } \\
\text { of City }\end{array}$} & \multirow{2}{*}{ Setting } & \multirow{2}{*}{$\begin{array}{c}\text { Description } \\
\text { of } \\
\text { Intervention }\end{array}$} & \multirow{2}{*}{$\begin{array}{l}\text { Intervention } \\
\text { Frequency }\end{array}$} & \multirow{2}{*}{ Outcomes } \\
\hline & & UC & RBPM & $\begin{array}{c}\text { Age } \\
\text { Interval }\end{array}$ & UC & RBPM & & & & & & & \\
\hline $\begin{array}{l}\text { Bosworth } \\
\text { (2007) [25] }\end{array}$ & $\begin{array}{c}\text { Treated } \\
\text { hypertensive } \\
\text { patients }\end{array}$ & 150 & 150 & $\begin{array}{l}\text { Child, } \\
\text { Adult, } \\
\text { Older } \\
\text { Adult }\end{array}$ & $\begin{array}{l}\text { Not re- } \\
\text { ported }\end{array}$ & $\begin{array}{l}\text { Not re- } \\
\text { ported }\end{array}$ & 18 & $\begin{array}{l}\text { Durham } \\
\text { (USA) }\end{array}$ & $\begin{array}{l}232,299 \text { in } \\
2005\end{array}$ & $\begin{array}{c}\text { Durham VA } \\
\text { general } \\
\text { internal } \\
\text { medicine } \\
\text { clinics } \\
\text { (Not } \\
\text { underserved) }\end{array}$ & $\begin{array}{c}\text { Nurse- } \\
\text { administered } \\
\text { tailored } \\
\text { behavioral } \\
\text { intervention } \\
\text { with } \\
\text { telemedicine } \\
\text { device } \\
\text { connected to } \\
\text { telephone }\end{array}$ & Once a day & $\begin{array}{c}\text { 1. Primary: } \\
\text { BP control. } \\
2 . \\
\text { Secondary: } \\
\text { knowledge } \\
\text { and } \\
\text { perceived } \\
\text { risks related } \\
\text { with hyper- } \\
\text { tension }\end{array}$ \\
\hline $\begin{array}{l}\text { Bosworth } \\
\text { (2007) [25] }\end{array}$ & $\begin{array}{c}\text { Treated } \\
\text { hypertensive } \\
\text { patients }\end{array}$ & 150 & 150 & $\begin{array}{l}\text { Child, } \\
\text { Adult, } \\
\text { Older } \\
\text { Adult }\end{array}$ & $\begin{array}{l}\text { Not re- } \\
\text { ported }\end{array}$ & $\begin{array}{l}\text { Not re- } \\
\text { ported }\end{array}$ & 18 & $\begin{array}{l}\text { Durham } \\
\text { (USA) }\end{array}$ & $\begin{array}{l}232,299 \text { in } \\
2005\end{array}$ & $\begin{array}{c}\text { Durham VA } \\
\text { general } \\
\text { internal } \\
\text { medicine } \\
\text { clinics } \\
\text { (Not } \\
\text { underserved) }\end{array}$ & $\begin{array}{c}\text { Nurse- } \\
\text { administered } \\
\text { medication } \\
\text { management }\end{array}$ & Once a day & $\begin{array}{c}\text { 1. Primary: } \\
\text { BP control } \\
2 . \\
\text { Secondary: } \\
\text { knowledge } \\
\text { and } \\
\text { perceived } \\
\text { risks related } \\
\text { with hyper- } \\
\text { tension }\end{array}$ \\
\hline $\begin{array}{l}\text { Bosworth } \\
\text { (2007) [25] }\end{array}$ & $\begin{array}{c}\text { Treated } \\
\text { hypertensive } \\
\text { patients }\end{array}$ & 150 & 150 & $\begin{array}{l}\text { Child, } \\
\text { Adult, } \\
\text { Older } \\
\text { Adult }\end{array}$ & $\begin{array}{l}\text { Not re- } \\
\text { ported }\end{array}$ & $\begin{array}{l}\text { Not re- } \\
\text { ported }\end{array}$ & 18 & $\begin{array}{l}\text { Durham } \\
\text { (USA) }\end{array}$ & $\begin{array}{l}232,299 \text { in } \\
2005\end{array}$ & $\begin{array}{c}\text { Durham VA } \\
\text { general } \\
\text { internal } \\
\text { medicine } \\
\text { clinics } \\
\text { (Not } \\
\text { underserved) }\end{array}$ & $\begin{array}{c}\text { Nurse- } \\
\text { administered } \\
\text { tailored } \\
\text { behavioral } \\
\text { intervention } \\
\text { and } \\
\text { medication } \\
\text { management }\end{array}$ & Once a day & $\begin{array}{c}\text { 1. Primary: } \\
\text { BP control } \\
2 . \\
\text { Secondary: } \\
\text { knowledge } \\
\text { and } \\
\text { perceived } \\
\text { risks related } \\
\text { with hyper- } \\
\text { tension }\end{array}$ \\
\hline
\end{tabular}


Table 1. Cont.

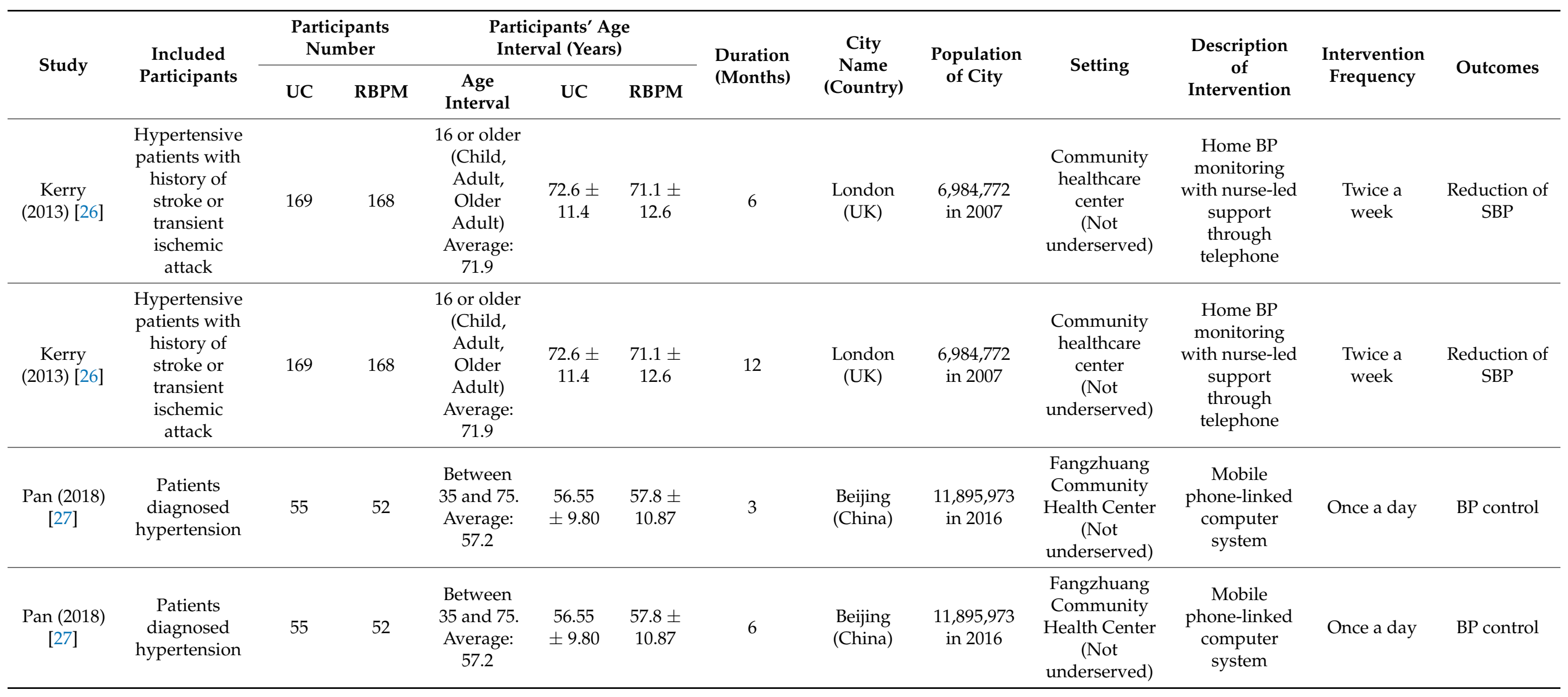


Table 1. Cont.

\begin{tabular}{|c|c|c|c|c|c|c|c|c|c|c|c|c|c|}
\hline \multirow{2}{*}{ Study } & \multirow{2}{*}{$\begin{array}{c}\text { Included } \\
\text { Participants }\end{array}$} & \multicolumn{2}{|c|}{$\begin{array}{l}\text { Participants } \\
\text { Number }\end{array}$} & \multicolumn{3}{|c|}{$\begin{array}{l}\text { Participants' Age } \\
\text { Interval (Years) }\end{array}$} & \multirow{2}{*}{$\begin{array}{l}\text { Duration } \\
\text { (Months) }\end{array}$} & \multirow{2}{*}{$\begin{array}{c}\text { City } \\
\text { Name } \\
\text { (Country) }\end{array}$} & \multirow{2}{*}{$\begin{array}{l}\text { Population } \\
\text { of City }\end{array}$} & \multirow{2}{*}{ Setting } & \multirow{2}{*}{$\begin{array}{l}\text { Description } \\
\text { of } \\
\text { Intervention }\end{array}$} & \multirow{2}{*}{$\begin{array}{c}\text { Intervention } \\
\text { Frequency }\end{array}$} & \multirow{2}{*}{ Outcomes } \\
\hline & & UC & RBPM & $\begin{array}{c}\text { Age } \\
\text { Interval }\end{array}$ & UC & RBPM & & & & & & & \\
\hline $\begin{array}{c}\text { Zha (2020) } \\
\text { [28] }\end{array}$ & $\begin{array}{l}\text { Uncontrolled } \\
\text { hypertensive } \\
\text { patients }\end{array}$ & 13 & 12 & $\begin{array}{c}\text { Between } \\
18 \text { and } 64 . \\
\text { Average: } \\
52.3\end{array}$ & $\begin{array}{c}55.5 \pm \\
5.2\end{array}$ & $\begin{array}{c}48.9 \pm \\
8.0\end{array}$ & 3 & $\begin{array}{c}\text { Newark } \\
\text { (USA) }\end{array}$ & $\begin{array}{l}278,366 \text { in } \\
2016\end{array}$ & $\begin{array}{c}\text { Jordan and } \\
\text { Harris } \\
\text { Community } \\
\text { Health Center } \\
\text { (Local } \\
\text { community } \\
\text { health center) } \\
\text { (Underserved) }\end{array}$ & $\begin{array}{l}\text { Smartphone- } \\
\text { linked system } \\
\text { by nurse }\end{array}$ & $\begin{array}{l}\text { Visit office } \\
\text { once a week. } \\
\text { Instant } \\
\text { feedback } \\
\text { after all mea- } \\
\text { surements. }\end{array}$ & $\begin{array}{l}\text { BP control } \\
\text { (Changes in } \\
\text { SBP and } \\
\text { DBP), } \\
\text { perceived } \\
\text { self-efficacy, } \\
\text { HRQOL }\end{array}$ \\
\hline $\begin{array}{c}\text { Zha (2020) } \\
\text { [28] }\end{array}$ & $\begin{array}{l}\text { Uncontrolled } \\
\text { hypertensive } \\
\text { patients }\end{array}$ & 13 & 12 & $\begin{array}{c}\text { Between } \\
18 \text { and } 64 . \\
\text { Average: } \\
52.3\end{array}$ & $\begin{array}{c}55.5 \pm \\
5.2\end{array}$ & $\begin{array}{c}48.9 \pm \\
8.0\end{array}$ & 6 & $\begin{array}{l}\text { Newark } \\
\text { (USA) }\end{array}$ & $\begin{array}{l}278,366 \text { in } \\
2016\end{array}$ & $\begin{array}{c}\text { Jordan and } \\
\text { Harris } \\
\text { Community } \\
\text { Health Center } \\
\text { (Local } \\
\text { community } \\
\text { health center) } \\
\text { (Underserved) }\end{array}$ & $\begin{array}{l}\text { Smartphone- } \\
\text { linked system } \\
\text { by nurse }\end{array}$ & $\begin{array}{l}\text { Visit office } \\
\text { once a week. } \\
\text { Instant } \\
\text { feedback } \\
\text { after all mea- } \\
\text { surements. }\end{array}$ & $\begin{array}{l}\text { BP control } \\
\text { (Changes in } \\
\text { SBP and } \\
\text { DBP), } \\
\text { perceived } \\
\text { self-efficacy, } \\
\text { HRQOL }\end{array}$ \\
\hline $\begin{array}{l}\text { Artinian } \\
\text { (2007) [29] }\end{array}$ & $\begin{array}{c}\text { African } \\
\text { American } \\
\text { hypertensive } \\
\text { patients }\end{array}$ & 157 & 164 & 18 or more & $\begin{array}{c}60.2 \pm \\
12.3\end{array}$ & $\begin{array}{c}59.1 \pm \\
13.0\end{array}$ & 3 & $\begin{array}{l}\text { Detroit } \\
\text { (USA) }\end{array}$ & $\begin{array}{l}594,562 \text { in } \\
2002\end{array}$ & $\begin{array}{c}\text { Family } \\
\text { community } \\
\text { center } \\
\text { (Underserved) }\end{array}$ & $\begin{array}{l}\text { Telephonic } \\
\text { transmission } \\
\text { with BP } \\
\text { monitoring } \\
\text { device linked } \\
\text { to telephone }\end{array}$ & Once a week & $\begin{array}{c}\text { Office BP } \\
\text { changes } \\
(\mathrm{SBP}, \mathrm{DBP})\end{array}$ \\
\hline $\begin{array}{l}\text { Artinian } \\
\text { (2007) [29] }\end{array}$ & $\begin{array}{c}\text { African } \\
\text { American } \\
\text { hypertensive } \\
\text { patients }\end{array}$ & 163 & 168 & 18 or more & $\begin{array}{c}60.2 \pm \\
12.3\end{array}$ & $\begin{array}{c}59.1 \pm \\
13.0\end{array}$ & 6 & $\begin{array}{l}\text { Detroit } \\
\text { (USA) }\end{array}$ & $\begin{array}{l}594,562 \text { in } \\
2002\end{array}$ & $\begin{array}{c}\text { Family } \\
\text { community } \\
\text { center } \\
\text { (Undeserved) }\end{array}$ & $\begin{array}{l}\text { Telephonic } \\
\text { transmission } \\
\text { with BP } \\
\text { monitoring } \\
\text { device linked } \\
\text { to telephone }\end{array}$ & $\begin{array}{l}\text { Once a } \\
\text { month }\end{array}$ & $\begin{array}{c}\text { Office BP } \\
\text { changes } \\
(\mathrm{SBP}, \mathrm{DBP})\end{array}$ \\
\hline
\end{tabular}


Table 1. Cont.

\begin{tabular}{|c|c|c|c|c|c|c|c|c|c|c|c|c|c|}
\hline \multirow{2}{*}{ Study } & \multirow{2}{*}{$\begin{array}{c}\text { Included } \\
\text { Participants }\end{array}$} & \multicolumn{2}{|c|}{$\begin{array}{l}\text { Participants } \\
\text { Number }\end{array}$} & \multicolumn{3}{|c|}{$\begin{array}{l}\text { Participants' Age } \\
\text { Interval (Years) }\end{array}$} & \multirow{2}{*}{$\begin{array}{l}\text { Duration } \\
\text { (Months) }\end{array}$} & \multirow{2}{*}{$\begin{array}{c}\text { City } \\
\text { Name } \\
\text { (Country) }\end{array}$} & \multirow{2}{*}{$\begin{array}{l}\text { Population } \\
\text { of City }\end{array}$} & \multirow{2}{*}{ Setting } & \multirow{2}{*}{$\begin{array}{c}\text { Description } \\
\text { of } \\
\text { Intervention }\end{array}$} & \multirow{2}{*}{$\begin{array}{l}\text { Intervention } \\
\text { Frequency }\end{array}$} & \multirow{2}{*}{ Outcomes } \\
\hline & & UC & RBPM & $\begin{array}{c}\text { Age } \\
\text { Interval }\end{array}$ & UC & RBPM & & & & & & & \\
\hline $\begin{array}{c}\text { Artinian } \\
\text { (2007) [29] }\end{array}$ & $\begin{array}{c}\text { African } \\
\text { American } \\
\text { hypertensive } \\
\text { patients }\end{array}$ & 169 & 167 & 18 or more & $\begin{array}{c}60.2 \pm \\
12.3\end{array}$ & $\begin{array}{c}59.1 \pm \\
13.0\end{array}$ & 12 & $\begin{array}{l}\text { Detroit } \\
\text { (USA) }\end{array}$ & $\begin{array}{c}594,562 \text { in } \\
2002\end{array}$ & $\begin{array}{c}\text { Family } \\
\text { community } \\
\text { center } \\
\text { (Undeserved) }\end{array}$ & $\begin{array}{c}\text { Telephonic } \\
\text { transmission } \\
\text { with BP } \\
\text { monitoring } \\
\text { device linked } \\
\text { to telephone }\end{array}$ & $\begin{array}{l}\text { Once a } \\
\text { month }\end{array}$ & $\begin{array}{c}\text { Office BP } \\
\text { changes } \\
(\mathrm{SBP}, \mathrm{DBP})\end{array}$ \\
\hline $\begin{array}{l}\text { Cicolini } \\
\text { (2013) [30] }\end{array}$ & $\begin{array}{c}\text { Treated or } \\
\text { untreated } \\
\text { hypertensive } \\
\text { patients }\end{array}$ & 98 & 100 & $\begin{array}{c}\text { Between } \\
18 \text { and } 80 . \\
\text { (Adult, } \\
\text { Older } \\
\text { Adult) } \\
\text { Average: } \\
59.1\end{array}$ & $\begin{array}{c}58.3 \pm \\
13.9\end{array}$ & $\begin{array}{c}59.8 \pm \\
15.0\end{array}$ & 3 & $\begin{array}{l}\text { Chieti } \\
\text { (Italy) }\end{array}$ & $\begin{array}{l}43,824 \text { in } \\
2011\end{array}$ & $\begin{array}{c}\text { Italian } \\
\text { Hypertension } \\
\text { Primary Care } \\
\text { Center } \\
\text { (Not } \\
\text { underserved) }\end{array}$ & $\begin{array}{l}\text { Nurse-led } \\
\text { reminder } \\
\text { through } \\
\text { e-mail }\end{array}$ & Once a week & $\begin{array}{l}\text { 1. BP } \\
\text { changes } \\
2 . \text { BMI, } \\
\text { alcohol con- } \\
\text { sumption, } \\
\text { cigarette } \\
\text { smoking, } \\
\text { adherence tc } \\
\text { therapy }\end{array}$ \\
\hline $\begin{array}{l}\text { Cicolini } \\
(2013)[30]\end{array}$ & $\begin{array}{c}\text { Treated or } \\
\text { untreated } \\
\text { hypertensive } \\
\text { patients }\end{array}$ & 98 & 100 & $\begin{array}{c}\text { Between } \\
18 \text { and } 80 . \\
\text { (Adult, } \\
\text { Older } \\
\text { Adult) } \\
\text { Average: } \\
59.1\end{array}$ & $\begin{array}{c}58.3 \pm \\
13.9\end{array}$ & $\begin{array}{c}59.8 \pm \\
15.0\end{array}$ & 6 & $\begin{array}{l}\text { Chieti } \\
\text { (Italy) }\end{array}$ & $\begin{array}{c}43,824 \text { in } \\
2011\end{array}$ & $\begin{array}{c}\text { Italian } \\
\text { Hypertension } \\
\text { Primary Care } \\
\text { Center } \\
\text { (Not } \\
\text { underserved) }\end{array}$ & $\begin{array}{c}\text { Nurse-led } \\
\text { reminder } \\
\text { through } \\
\text { e-mail }\end{array}$ & Once a week & $\begin{array}{c}\text { 1. BP } \\
\text { changes } \\
2 . \text { BMI, } \\
\text { alcohol con- } \\
\text { sumption, } \\
\text { cigarette } \\
\text { smoking, } \\
\text { adherence to } \\
\text { therapy }\end{array}$ \\
\hline
\end{tabular}


Table 1. Cont.

\begin{tabular}{|c|c|c|c|c|c|c|c|c|c|c|c|c|c|}
\hline \multirow{2}{*}{ Study } & \multirow{2}{*}{$\begin{array}{c}\text { Included } \\
\text { Participants }\end{array}$} & \multicolumn{2}{|c|}{$\begin{array}{l}\text { Participants } \\
\text { Number }\end{array}$} & \multicolumn{3}{|c|}{$\begin{array}{l}\text { Participants' Age } \\
\text { Interval (Years) }\end{array}$} & \multirow{2}{*}{$\begin{array}{l}\text { Duration } \\
\text { (Months) }\end{array}$} & \multirow{2}{*}{$\begin{array}{c}\text { City } \\
\text { Name } \\
\text { (Country) }\end{array}$} & \multirow{2}{*}{$\begin{array}{l}\text { Population } \\
\text { of City }\end{array}$} & \multirow{2}{*}{ Setting } & \multirow{2}{*}{$\begin{array}{c}\text { Description } \\
\text { of } \\
\text { Intervention }\end{array}$} & \multirow{2}{*}{$\begin{array}{l}\text { Intervention } \\
\text { Frequency }\end{array}$} & \multirow{2}{*}{ Outcomes } \\
\hline & & UC & RBPM & $\begin{array}{c}\text { Age } \\
\text { Interval }\end{array}$ & $\mathrm{UC}$ & RBPM & & & & & & & \\
\hline $\begin{array}{l}\text { Hebert } \\
\text { (2012) [31] }\end{array}$ & $\begin{array}{l}\text { Uncontrolled } \\
\text { hypertensive } \\
\text { patients }\end{array}$ & 83 & 85 & $\begin{array}{c}18 \text { or more. } \\
\text { Average: } \\
60.8\end{array}$ & $\begin{array}{c}(61.3 \pm \\
11.7)\end{array}$ & $\begin{array}{c}61.3 \pm \\
11.7\end{array}$ & 9 & $\begin{array}{l}\text { New York } \\
\text { (USA) }\end{array}$ & $\begin{array}{c}8,174,959 \\
\text { in } 2010\end{array}$ & $\begin{array}{c}\text { One academic } \\
\text { medical center, } \\
\text { two } \\
\text { medium-sized } \\
\text { hospitals, one } \\
\text { community } \\
\text { hospital } \\
\text { (Under- } \\
\text { served) }\end{array}$ & Telephone & $\begin{array}{l}\text { Once a week } \\
\text { (Meetings: } \\
\text { once in two } \\
\text { weeks) }\end{array}$ & $\begin{array}{l}\text { Blood } \\
\text { pressure } \\
\text { reduction }\end{array}$ \\
\hline $\begin{array}{c}\text { Hebert } \\
(2012)[31]\end{array}$ & $\begin{array}{l}\text { Uncontrolled } \\
\text { hypertensive } \\
\text { patients }\end{array}$ & 78 & 79 & $\begin{array}{c}18 \text { or more. } \\
\text { Average: } \\
60.8 \\
\text { Average: } \\
60.8\end{array}$ & $\begin{array}{c}(61.3 \pm \\
11.7)\end{array}$ & $\begin{array}{c}61.3 \pm \\
11.7\end{array}$ & 18 & $\begin{array}{l}\text { New York } \\
\text { (USA) }\end{array}$ & $\begin{array}{c}7,721,457 \\
\text { in } 2010\end{array}$ & $\begin{array}{c}\text { One academic } \\
\text { medical center, } \\
\text { two } \\
\text { medium-sized } \\
\text { hospitals, one } \\
\text { community } \\
\text { hospital } \\
\text { (Under- } \\
\text { served) }\end{array}$ & Telephone & $\begin{array}{l}\text { Once a week } \\
\text { (Meetings: } \\
\text { once in two } \\
\text { weeks) }\end{array}$ & $\begin{array}{l}\text { Blood } \\
\text { pressure } \\
\text { reduction }\end{array}$ \\
\hline $\begin{array}{c}\text { Kim (2014) } \\
{[32]}\end{array}$ & $\begin{array}{l}\text { Uncontrolled } \\
\text { Korean- } \\
\text { American } \\
\text { hypertensive } \\
\text { seniors }\end{array}$ & 192 & 191 & $\begin{array}{l}60 \text { or older } \\
\text { adult. } \\
\text { Average: } \\
70.9\end{array}$ & $\begin{array}{c}71.2 \pm \\
5.6\end{array}$ & $\begin{array}{c}70.6 \pm \\
5.0\end{array}$ & 6 & $\begin{array}{c}\text { Ellicott } \\
\text { City (USA) }\end{array}$ & $\begin{array}{l}60,489 \text { in } \\
2007\end{array}$ & $\begin{array}{c}\text { Korean } \\
\text { Resource } \\
\text { Center } \\
\text { (Hospital) } \\
\text { (Not } \\
\text { Undeserved) }\end{array}$ & $\begin{array}{l}\text { Telephone- } \\
\text { monitoring } \\
\text { system and } \\
\text { telephone } \\
\text { counseling }\end{array}$ & $\begin{array}{c}\text { At least once } \\
\text { a week } \\
\text { (Measure- } \\
\text { ment: at } \\
\text { least twice a } \\
\text { day, } \\
\text { Monthly } \\
\text { telephone } \\
\text { counseling) }\end{array}$ & $\begin{array}{l}\text { Changes in } \\
\text { SBP and } \\
\text { DBP }\end{array}$ \\
\hline
\end{tabular}


Table 1. Cont.

\begin{tabular}{|c|c|c|c|c|c|c|c|c|c|c|c|c|c|}
\hline \multirow{2}{*}{ Study } & \multirow{2}{*}{$\begin{array}{c}\text { Included } \\
\text { Participants }\end{array}$} & \multicolumn{2}{|c|}{$\begin{array}{l}\text { Participants } \\
\text { Number }\end{array}$} & \multicolumn{3}{|c|}{$\begin{array}{l}\text { Participants' Age } \\
\text { Interval (Years) }\end{array}$} & \multirow{2}{*}{$\begin{array}{l}\text { Duration } \\
\text { (Months) }\end{array}$} & \multirow{2}{*}{$\begin{array}{c}\text { City } \\
\text { Name } \\
\text { (Country) }\end{array}$} & \multirow{2}{*}{$\begin{array}{l}\text { Population } \\
\text { of City }\end{array}$} & \multirow{2}{*}{ Setting } & \multirow{2}{*}{$\begin{array}{l}\text { Description } \\
\text { of } \\
\text { Intervention }\end{array}$} & \multirow{2}{*}{$\begin{array}{c}\text { Intervention } \\
\text { Frequency }\end{array}$} & \multirow{2}{*}{ Outcomes } \\
\hline & & UC & RBPM & $\begin{array}{c}\text { Age } \\
\text { Interval }\end{array}$ & UC & RBPM & & & & & & & \\
\hline $\begin{array}{c}\text { Kim (2014) } \\
{[32]}\end{array}$ & $\begin{array}{l}\text { Uncontrolled } \\
\text { Korean- } \\
\text { American } \\
\text { hypertensive } \\
\text { seniors }\end{array}$ & 185 & 187 & $\begin{array}{l}60 \text { or older } \\
\text { adult. } \\
\text { Average: } \\
70.9\end{array}$ & $\begin{array}{c}71.2 \pm \\
5.6\end{array}$ & $\begin{array}{c}70.6 \pm \\
5.0\end{array}$ & 12 & $\begin{array}{c}\text { Ellicott } \\
\text { City (USA) }\end{array}$ & $\begin{array}{l}60,489 \text { in } \\
2007\end{array}$ & $\begin{array}{c}\text { Korean } \\
\text { Resource } \\
\text { Center } \\
\text { (Hospital) } \\
\text { (Not } \\
\text { Undeserved) }\end{array}$ & $\begin{array}{l}\text { Telephone- } \\
\text { monitoring } \\
\text { system and } \\
\text { telephone } \\
\text { counseling }\end{array}$ & $\begin{array}{l}\text { At least once } \\
\text { a week } \\
\text { (Measure- } \\
\text { ment: at } \\
\text { least twice a } \\
\text { day, } \\
\text { Monthly } \\
\text { telephone } \\
\text { counseling, }\end{array}$ & $\begin{array}{l}\text { Changes in } \\
\text { SBP and } \\
\text { DBP }\end{array}$ \\
\hline $\begin{array}{c}\text { Kim (2014) } \\
{[32]}\end{array}$ & $\begin{array}{l}\text { Uncontrolled } \\
\text { Korean- } \\
\text { American } \\
\text { hypertensive } \\
\text { seniors }\end{array}$ & 185 & 184 & $\begin{array}{l}60 \text { or older } \\
\text { adult. } \\
\text { Average: } \\
70.9\end{array}$ & $\begin{array}{c}71.2 \pm \\
5.6\end{array}$ & $\begin{array}{c}70.6 \pm \\
5.0\end{array}$ & 18 & $\begin{array}{c}\text { Ellicott } \\
\text { City (USA) }\end{array}$ & $\begin{array}{l}60,489 \text { in } \\
2007\end{array}$ & $\begin{array}{c}\text { Korean } \\
\text { Resource } \\
\text { Center } \\
\text { (Hospital) } \\
\text { (Not } \\
\text { Undeserved) }\end{array}$ & $\begin{array}{l}\text { Telephone- } \\
\text { monitoring } \\
\text { system and } \\
\text { telephone } \\
\text { counseling }\end{array}$ & $\begin{array}{c}\text { At least once } \\
\text { a week } \\
\text { (Measure- } \\
\text { ment: at } \\
\text { least twice a } \\
\text { day, } \\
\text { Monthly } \\
\text { telephone } \\
\text { counseling, }\end{array}$ & $\begin{array}{l}\text { Changes in } \\
\text { SBP and } \\
\text { DBP }\end{array}$ \\
\hline $\begin{array}{l}\text { Mohsen } \\
(2020)[33]\end{array}$ & $\begin{array}{l}\text { Treated } \\
\text { hypertensive } \\
\text { patients with } \\
\text { antihyperten- } \\
\text { sive } \\
\text { medication }\end{array}$ & 50 & 50 & $\begin{array}{c}\text { Between } \\
35 \text { and } 65 . \\
\text { Average: } \\
56.41\end{array}$ & $\begin{array}{l}55.01 \\
\pm 7.50\end{array}$ & $\begin{array}{l}57.81 \\
\pm 9.52\end{array}$ & 3 & $\begin{array}{l}\text { Shibin El } \\
\text { Kom } \\
\text { (Egypt) }\end{array}$ & $\begin{array}{l}190.064 \text { in } \\
2019\end{array}$ & $\begin{array}{c}\text { Medical } \\
\text { outpatient } \\
\text { clinic of } \\
\text { Menoufia } \\
\text { University } \\
\text { Hospital } \\
\text { (Not } \\
\text { Undeserved) }\end{array}$ & $\begin{array}{c}\text { Tele-nursing } \\
\text { intervention } \\
\text { with } \\
\text { telephone } \\
\text { support }\end{array}$ & $\begin{array}{c}\text { Twice a } \\
\text { week (Mea- } \\
\text { surement: } \\
\text { every day) }\end{array}$ & $\begin{array}{l}\text { 1. Reduction } \\
\text { of SBP and } \\
\text { DBP } \\
\text { 2. BMI } \\
\text { difference }\end{array}$ \\
\hline
\end{tabular}


Table 1. Cont.

\begin{tabular}{|c|c|c|c|c|c|c|c|c|c|c|c|c|c|}
\hline \multirow{2}{*}{ Study } & \multirow{2}{*}{$\begin{array}{c}\text { Included } \\
\text { Participants }\end{array}$} & \multicolumn{2}{|c|}{$\begin{array}{l}\text { Participants } \\
\text { Number }\end{array}$} & \multicolumn{3}{|c|}{$\begin{array}{l}\text { Participants' Age } \\
\text { Interval (Years) }\end{array}$} & \multirow{2}{*}{$\begin{array}{l}\text { Duration } \\
\text { (Months) }\end{array}$} & \multirow{2}{*}{$\begin{array}{c}\text { City } \\
\text { Name } \\
\text { (Country) }\end{array}$} & \multirow{2}{*}{$\begin{array}{c}\text { Population } \\
\text { of City }\end{array}$} & \multirow{2}{*}{ Setting } & \multirow{2}{*}{$\begin{array}{c}\text { Description } \\
\text { of } \\
\text { Intervention }\end{array}$} & \multirow{2}{*}{$\begin{array}{l}\text { Intervention } \\
\text { Frequency }\end{array}$} & \multirow{2}{*}{ Outcomes } \\
\hline & & UC & RBPM & $\begin{array}{c}\text { Age } \\
\text { Interval }\end{array}$ & UC & RBPM & & & & & & & \\
\hline $\begin{array}{l}\text { Mohsen } \\
\text { (2020) [33] }\end{array}$ & $\begin{array}{c}\text { Treated } \\
\text { hypertensive } \\
\text { patients with } \\
\text { medication }\end{array}$ & 50 & 50 & $\begin{array}{c}\text { Between } \\
35 \text { and } 65 . \\
\text { Average: } \\
56.41\end{array}$ & $\begin{array}{l}55.01 \\
\pm 7.50\end{array}$ & $\begin{array}{l}57.81 \\
\pm 9.52\end{array}$ & 6 & $\begin{array}{l}\text { Shibin El } \\
\text { Kom } \\
\text { (Egypt) }\end{array}$ & $\begin{array}{l}190.064 \text { in } \\
2019\end{array}$ & $\begin{array}{c}\text { Medical } \\
\text { outpatient } \\
\text { clinic of } \\
\text { Menoufia } \\
\text { University } \\
\text { Hospital } \\
\text { (Not } \\
\text { Undeserved) }\end{array}$ & $\begin{array}{c}\text { Tele-nursing } \\
\text { intervention } \\
\text { with } \\
\text { telephone } \\
\text { support }\end{array}$ & $\begin{array}{l}\text { Twice a } \\
\text { week. (Mea- } \\
\text { surement: } \\
\text { every day) }\end{array}$ & $\begin{array}{l}\text { 1. Reduction } \\
\text { of SBP and } \\
\text { DBP } \\
\text { 2. BMI } \\
\text { difference }\end{array}$ \\
\hline $\begin{array}{c}\text { Pour } \\
\text { (2020) [34] }\end{array}$ & $\begin{array}{c}\text { Treated } \\
\text { hypertensive } \\
\text { patients with } \\
\text { medication }\end{array}$ & 21 & 21 & $\begin{array}{c}\text { Between } \\
35 \text { and } 64 . \\
\text { Average: } \\
55.7\end{array}$ & $\begin{array}{l}56.71 \\
\pm 5.73\end{array}$ & $\begin{array}{l}54.71 \\
\pm 6.11\end{array}$ & 3 & $\begin{array}{l}\text { Tehran } \\
\text { (Iran) }\end{array}$ & $\begin{array}{c}7,250,693 \\
\text { in } 2019\end{array}$ & $\begin{array}{c}\text { Military } \\
\text { hospital } \\
\text { (Not } \\
\text { underserved) }\end{array}$ & $\begin{array}{l}\text { Interactive } \\
\text { SMS }\end{array}$ & Once a week & $\begin{array}{l}\text { BP control } \\
\text { (Changes in } \\
\text { SBP and } \\
\text { DBP), }\end{array}$ \\
\hline $\begin{array}{c}\text { Pour } \\
\text { (2020) [34] }\end{array}$ & $\begin{array}{c}\text { Treated } \\
\text { hypertensive } \\
\text { patients with } \\
\text { medication }\end{array}$ & 21 & 21 & $\begin{array}{c}\text { Between } \\
35 \text { and } 64 . \\
\text { Average: } \\
55.7\end{array}$ & $\begin{array}{l}56.71 \\
\pm 5.73\end{array}$ & $\begin{array}{l}54.71 \\
\pm 6.11\end{array}$ & 4 & $\begin{array}{l}\text { Tehran } \\
\text { (Iran) }\end{array}$ & $\begin{array}{c}7,250,693 \\
\text { in } 2019\end{array}$ & $\begin{array}{c}\text { Military } \\
\text { hospital } \\
\text { (Not } \\
\text { underserved) }\end{array}$ & $\begin{array}{l}\text { Interactive } \\
\text { SMS }\end{array}$ & Once a week & $\begin{array}{l}\text { BP control } \\
\text { (Changes in } \\
\text { SBP and } \\
\text { DBP), }\end{array}$ \\
\hline $\begin{array}{c}\text { Pour } \\
\text { (2020) [34] }\end{array}$ & $\begin{array}{c}\text { Treated } \\
\text { hypertensive } \\
\text { patients with } \\
\text { medication }\end{array}$ & 21 & 21 & $\begin{array}{c}\text { Between } \\
35 \text { and } 64 . \\
\text { Average: } \\
55.7\end{array}$ & $\begin{array}{c}56.71 \\
\pm 5.73\end{array}$ & $\begin{array}{l}54.71 \\
\pm 6.11\end{array}$ & 3 & $\begin{array}{l}\text { Tehran } \\
\text { (Iran) }\end{array}$ & $\begin{array}{l}7,250,693 \\
\text { in } 2019\end{array}$ & $\begin{array}{c}\text { Military } \\
\text { hospital } \\
\text { (Not } \\
\text { underserved) }\end{array}$ & $\begin{array}{l}\text { Non- } \\
\text { Interactive } \\
\text { SMS }\end{array}$ & Once a week & $\begin{array}{l}\text { BP control } \\
\text { (Changes in } \\
\text { SBP and } \\
\text { DBP), }\end{array}$ \\
\hline $\begin{array}{c}\text { Pour } \\
(2020)[34]\end{array}$ & $\begin{array}{c}\text { Treated } \\
\text { hypertensive } \\
\text { patients with } \\
\text { medication }\end{array}$ & 21 & 21 & $\begin{array}{c}\text { Between } \\
35 \text { and } 64 . \\
\text { Average: } \\
55.7\end{array}$ & $\begin{array}{l}56.71 \\
\pm 5.73\end{array}$ & $\begin{array}{l}54.71 \\
\pm 6.11\end{array}$ & 4 & $\begin{array}{l}\text { Tehran } \\
\text { (Iran) }\end{array}$ & $\begin{array}{c}7,250,693 \\
\text { in } 2019\end{array}$ & $\begin{array}{c}\text { Military } \\
\text { hospital } \\
\text { (Not } \\
\text { underserved) }\end{array}$ & $\begin{array}{l}\text { Non- } \\
\text { Interactive } \\
\text { SMS }\end{array}$ & Once a week & $\begin{array}{l}\text { BP control } \\
\text { (Changes in } \\
\text { SBP and } \\
\text { DBP), }\end{array}$ \\
\hline
\end{tabular}


Table 1. Cont.

\begin{tabular}{|c|c|c|c|c|c|c|c|c|c|c|c|c|c|}
\hline \multirow{2}{*}{ Study } & \multirow{2}{*}{$\begin{array}{c}\text { Included } \\
\text { Participants }\end{array}$} & \multicolumn{2}{|c|}{$\begin{array}{l}\text { Participants } \\
\text { Number }\end{array}$} & \multicolumn{3}{|c|}{$\begin{array}{l}\text { Participants' Age } \\
\text { Interval (Years) }\end{array}$} & \multirow{2}{*}{$\begin{array}{l}\text { Duration } \\
\text { (Months) }\end{array}$} & \multirow{2}{*}{$\begin{array}{c}\text { City } \\
\text { Name } \\
\text { (Country) }\end{array}$} & \multirow{2}{*}{$\begin{array}{l}\text { Population } \\
\text { of City }\end{array}$} & \multirow{2}{*}{ Setting } & \multirow{2}{*}{$\begin{array}{c}\text { Description } \\
\text { of } \\
\text { Intervention }\end{array}$} & \multirow{2}{*}{$\begin{array}{c}\text { Intervention } \\
\text { Frequency }\end{array}$} & \multirow{2}{*}{ Outcomes } \\
\hline & & UC & RBPM & $\begin{array}{c}\text { Age } \\
\text { Interval }\end{array}$ & UC & RBPM & & & & & & & \\
\hline $\begin{array}{l}\text { Rubinstein } \\
\text { (2016) [35] }\end{array}$ & $\begin{array}{l}\text { Untreated pre- } \\
\text { hypertensive } \\
\text { patients }\end{array}$ & 276 & 270 & $\begin{array}{c}\text { Between } \\
30 \text { and } 60 . \\
\text { Average: } \\
43.4\end{array}$ & $\begin{array}{c}43.2 \pm \\
8.4\end{array}$ & $\begin{array}{c}43.6 \pm \\
8.4\end{array}$ & 6 & $\begin{array}{l}\text { Buenos } \\
\text { Aires (Ar- } \\
\text { gentina) } \\
\text { and } \\
\text { Guatemala } \\
\text { City } \\
\text { (Guatemala) } \\
\text { and Lima } \\
\text { (Peru) }\end{array}$ & $\begin{array}{c}\text { 12,271,254 } \\
\text { (Buenos } \\
\text { Aires) and } \\
880,893 \\
\text { (Guatemala } \\
\text { City) and } \\
\text { 7,136,586 } \\
\text { (Lima) in } \\
2012\end{array}$ & $\begin{array}{c}\text { Institute for } \\
\text { Clinical } \\
\text { Effectiveness } \\
\text { and Health } \\
\text { Policy } \\
\text { (Buenos Aires, } \\
\text { Argentina), } \\
\text { Institute of } \\
\text { Nutrition of } \\
\text { Central } \\
\text { America and } \\
\text { Panama } \\
\text { (Guatemala } \\
\text { City, } \\
\text { Guatemala), } \\
\text { Universidad } \\
\text { Peruana } \\
\text { Cayetano } \\
\text { Heredia } \\
\text { (Lima, Peru) } \\
\text { (Underserved) }\end{array}$ & $\begin{array}{l}\text { Mobile phone } \\
\text { transmission }\end{array}$ & $\begin{array}{l}\text { Once a } \\
\text { month }\end{array}$ & $\begin{array}{l}\text { Mean } \\
\text { changes in } \\
\text { SBP and } \\
\text { DBP }\end{array}$ \\
\hline
\end{tabular}


Table 1. Cont.

\begin{tabular}{|c|c|c|c|c|c|c|c|c|c|c|c|c|c|}
\hline \multirow{2}{*}{ Study } & \multirow{2}{*}{$\begin{array}{c}\text { Included } \\
\text { Participants }\end{array}$} & \multicolumn{2}{|c|}{$\begin{array}{l}\text { Participants } \\
\text { Number }\end{array}$} & \multicolumn{3}{|c|}{$\begin{array}{l}\text { Participants' Age } \\
\text { Interval (Years) }\end{array}$} & \multirow{2}{*}{$\begin{array}{l}\text { Duration } \\
\text { (Months) }\end{array}$} & \multirow{2}{*}{$\begin{array}{c}\text { City } \\
\text { Name } \\
\text { (Country) }\end{array}$} & \multirow{2}{*}{$\begin{array}{l}\text { Population } \\
\text { of City }\end{array}$} & \multirow{2}{*}{ Setting } & \multirow{2}{*}{$\begin{array}{c}\text { Description } \\
\text { of } \\
\text { Intervention }\end{array}$} & \multirow{2}{*}{$\begin{array}{l}\text { Intervention } \\
\text { Frequency }\end{array}$} & \multirow{2}{*}{ Outcomes } \\
\hline & & UC & RBPM & $\begin{array}{c}\text { Age } \\
\text { Interval }\end{array}$ & UC & RBPM & & & & & & & \\
\hline $\begin{array}{l}\text { Rubinstein } \\
(2016)[35]\end{array}$ & $\begin{array}{l}\text { Untreated pre- } \\
\text { hypertensive } \\
\text { patients }\end{array}$ & 287 & 266 & $\begin{array}{c}\text { Between } \\
30 \text { and } 60 . \\
\text { Average: } \\
43.4\end{array}$ & $\begin{array}{c}43.2 \pm \\
8.4\end{array}$ & $\begin{array}{c}43.6 \pm \\
8.4\end{array}$ & 12 & $\begin{array}{l}\text { Buenos } \\
\text { Aires (Ar- } \\
\text { gentina) } \\
\text { and } \\
\text { Guatemala } \\
\text { City } \\
\text { (Guatemala) } \\
\text { and Lima } \\
\text { (Peru) }\end{array}$ & $\begin{array}{l}\text { 12,271,254 } \\
\text { (Buenos } \\
\text { Aires) and } \\
880,893 \\
\text { (Guatemala } \\
\text { city) and } \\
7,136,586 \\
\text { (Lima) in } \\
2012\end{array}$ & $\begin{array}{c}\text { Institute for } \\
\text { Clinical } \\
\text { Effectiveness } \\
\text { and Health } \\
\text { Policy } \\
\text { (Buenos Aires, } \\
\text { Argentina), } \\
\text { Insitute of } \\
\text { Nutrition of } \\
\text { Central } \\
\text { America and } \\
\text { Panama } \\
\text { (Guatemala } \\
\text { City, } \\
\text { Guatemala), } \\
\text { Universidad } \\
\text { Peruana } \\
\text { Cayetano } \\
\text { Heredia } \\
\text { (Lima, Peru) } \\
\text { (Underserved) }\end{array}$ & $\begin{array}{l}\text { Mobile phone } \\
\text { transmission }\end{array}$ & $\begin{array}{l}\text { Once a } \\
\text { month }\end{array}$ & $\begin{array}{l}\text { Mean } \\
\text { changes in } \\
\text { SBP and } \\
\text { DBP }\end{array}$ \\
\hline $\begin{array}{l}\text { Hill (1999) } \\
\text { [41] }\end{array}$ & $\begin{array}{l}\text { Black or } \\
\text { African } \\
\text { American } \\
\text { hypertensive } \\
\text { young male } \\
\text { residents } \\
\text { within } \\
\text { hospital } \\
\text { catchment } \\
\text { area }\end{array}$ & 77 & 78 & $\begin{array}{r}\text { Betw } \\
\text { Av }\end{array}$ & $\begin{array}{l}\text { en } 22 \text { and } \\
\text { rage: } 39.0\end{array}$ & & 12 & $\begin{array}{l}\text { Baltimore } \\
\text { (USA) }\end{array}$ & $\begin{array}{c}503,998 \text { in } \\
1995\end{array}$ & $\begin{array}{c}\text { Johns } \\
\text { Hopkins } \\
\text { Hospital } \\
\text { Outpatient } \\
\text { General } \\
\text { Clinical } \\
\text { Research } \\
\text { Center } \\
\text { (Underserved) }\end{array}$ & Telephone & $\begin{array}{l}\text { Once a } \\
\text { month }\end{array}$ & $\begin{array}{l}\text { Office BP } \\
\text { changes }\end{array}$ \\
\hline
\end{tabular}


Table 1. Cont.

\begin{tabular}{|c|c|c|c|c|c|c|c|c|c|c|c|c|c|}
\hline \multirow{2}{*}{ Study } & \multirow{2}{*}{$\begin{array}{c}\text { Included } \\
\text { Participants }\end{array}$} & \multicolumn{2}{|c|}{$\begin{array}{l}\text { Participants } \\
\text { Number }\end{array}$} & \multicolumn{3}{|c|}{$\begin{array}{l}\text { Participants' Age } \\
\text { Interval (Years) }\end{array}$} & \multirow{2}{*}{$\begin{array}{l}\text { Duration } \\
\text { (Months) }\end{array}$} & \multirow{2}{*}{$\begin{array}{c}\text { City } \\
\text { Name } \\
\text { (Country) }\end{array}$} & \multirow{2}{*}{$\begin{array}{l}\text { Population } \\
\text { of City }\end{array}$} & \multirow{2}{*}{ Setting } & \multirow{2}{*}{$\begin{array}{l}\text { Description } \\
\text { of } \\
\text { Intervention }\end{array}$} & \multirow{2}{*}{$\begin{array}{l}\text { Intervention } \\
\text { Frequency }\end{array}$} & \multirow{2}{*}{ Outcomes } \\
\hline & & UC & RBPM & $\begin{array}{c}\text { Age } \\
\text { Interval }\end{array}$ & UC & RBPM & & & & & & & \\
\hline $\begin{array}{l}\text { Friedman } \\
\text { (1996) [42] }\end{array}$ & $\begin{array}{c}\text { Treated } \\
\text { hypertensive } \\
\text { patients }\end{array}$ & 134 & 133 & $\begin{array}{c}\text { Over } 60 \\
\text { Average: } \\
76.5\end{array}$ & 77 & 76 & 6 & $\begin{array}{l}\text { Boston } \\
\text { (USA) }\end{array}$ & $\begin{array}{c}534,743 \text { in } \\
1994\end{array}$ & $\begin{array}{c}\text { Senior centers } \\
\text { in } 29 \text { different } \\
\text { communi- } \\
\text { ties(Not } \\
\text { underserved) }\end{array}$ & $\begin{array}{l}\text { Telephone- } \\
\text { linked } \\
\text { computer } \\
\text { system }\end{array}$ & Once a week & $\begin{array}{l}\text { Office BP } \\
\text { changes }\end{array}$ \\
\hline $\begin{array}{l}\text { McMahon } \\
\text { (2005) [43] }\end{array}$ & $\begin{array}{c}\text { Poorly } \\
\text { controlled } \\
\text { diabetics and } \\
\text { hypertensive } \\
\text { patients }\end{array}$ & 35 & 37 & $\begin{array}{c}\text { Older than } \\
18 . \\
\text { Average: } \\
63.5\end{array}$ & $63 \pm 7$ & $64 \pm 7$ & 12 & $\begin{array}{l}\text { Boston } \\
\text { (USA) }\end{array}$ & $\begin{array}{l}580,352 \text { in } \\
2001\end{array}$ & $\begin{array}{c}\text { Hospital } \\
\text { (Not } \\
\text { underserved) }\end{array}$ & Web-base & $\begin{array}{c}\text { At least } \\
\text { three times a } \\
\text { week }\end{array}$ & $\begin{array}{c}\text { Changes in } \\
\mathrm{A}_{1 \mathrm{c}}, \mathrm{BP}, \\
\text { lipid } \\
\text { profiles }\end{array}$ \\
\hline $\begin{array}{c}\text { Shea (2006) } \\
{[44]}\end{array}$ & $\begin{array}{c}\text { Diabetic } \\
\text { hypertensive } \\
\text { patients }\end{array}$ & 347 & 333 & $\begin{array}{c}55 \text { or older } \\
\text { (Adult, } \\
\text { Older } \\
\text { Adult) } \\
\text { Average: } \\
70.8 \pm 6.7\end{array}$ & $\begin{array}{c}70.9 \pm \\
6.8\end{array}$ & $\begin{array}{c}70.8 \pm \\
6.5\end{array}$ & 12 & $\begin{array}{l}\text { Syracuse } \\
\text { (USA) }\end{array}$ & $\begin{array}{l}129,966 \text { in } \\
2005\end{array}$ & $\begin{array}{c}\text { SUNY } \\
\text { Upstate } \\
\text { Medical } \\
\text { University } \\
\text { hospital, } \\
\text { (Underserved) }\end{array}$ & $\begin{array}{l}\text { Telephone- } \\
\text { linked web } \\
\text { system }\end{array}$ & Regularly & $\begin{array}{c}\text { Changes in } \\
\text { hemoglobin } \\
\mathrm{A}_{1 \mathrm{c}}, \mathrm{BP}, \\
\text { cholesterol } \\
\text { level }\end{array}$ \\
\hline $\begin{array}{l}\text { Carrasco } \\
(2008)[45]\end{array}$ & $\begin{array}{c}\text { Treated or } \\
\text { untreated } \\
\text { hypertensive } \\
\text { patients }\end{array}$ & 142 & 131 & $\begin{array}{l}\text { Average } \\
\text { age: } 62.5\end{array}$ & $\begin{array}{c}62.8 \pm \\
12.5\end{array}$ & $\begin{array}{c}62.1 \pm \\
11.9\end{array}$ & 3 & $\begin{array}{l}\text { Madrid } \\
\text { (Spain) }\end{array}$ & $\begin{array}{c}3,116,909 \\
\text { in } 2006\end{array}$ & $\begin{array}{l}21 \text { regional } \\
\text { public health } \\
\text { centers (the } \\
\text { corporative } \\
\text { network of the } \\
\text { "Servicio } \\
\text { Madrileno de } \\
\text { Salud") } \\
\text { (Not } \\
\text { underserved) }\end{array}$ & $\begin{array}{l}\text { Mobile phone } \\
\text { transmission }\end{array}$ & $\begin{array}{l}\text { During the } \\
\text { six-month } \\
\text { follow-up, } \\
\text { four times a } \\
\text { week } \\
\text { (Monday } \\
\text { and } \\
\text { Thursday, } \\
\text { morning } \\
\text { and night) }\end{array}$ & $\begin{array}{l}\text { 1. BP } \\
\text { control } \\
2 . \text { the } \\
\text { impact on } \\
\text { patient QoL } \\
\text { and anxiety, } \\
\text { and } \\
\text { economic } \\
\text { aspects } \\
\text { concerning } \\
\text { the viability } \\
\text { of the } \\
\text { telemedicine } \\
\text { system }\end{array}$ \\
\hline
\end{tabular}


Table 1. Cont.

\begin{tabular}{|c|c|c|c|c|c|c|c|c|c|c|c|c|c|}
\hline \multirow{2}{*}{ Study } & \multirow{2}{*}{$\begin{array}{c}\text { Included } \\
\text { Participants }\end{array}$} & \multicolumn{2}{|c|}{$\begin{array}{l}\text { Participants } \\
\text { Number }\end{array}$} & \multicolumn{3}{|c|}{$\begin{array}{l}\text { Participants' Age } \\
\text { Interval (Years) }\end{array}$} & \multirow{2}{*}{$\begin{array}{l}\text { Duration } \\
\text { (Months) }\end{array}$} & \multirow{2}{*}{$\begin{array}{c}\text { City } \\
\text { Name } \\
\text { (Country) }\end{array}$} & \multirow{2}{*}{$\begin{array}{l}\text { Population } \\
\text { of City }\end{array}$} & \multirow{2}{*}{ Setting } & \multirow{2}{*}{$\begin{array}{c}\text { Description } \\
\text { of } \\
\text { Intervention }\end{array}$} & \multirow{2}{*}{$\begin{array}{c}\text { Intervention } \\
\text { Frequency }\end{array}$} & \multirow{2}{*}{ Outcomes } \\
\hline & & UC & RBPM & $\begin{array}{c}\text { Age } \\
\text { Interval }\end{array}$ & UC & RBPM & & & & & & & \\
\hline $\begin{array}{c}\text { Green } \\
(2008)[46]\end{array}$ & $\begin{array}{c}\text { Treated } \\
\text { hypertensive } \\
\text { patients }\end{array}$ & 247 & 246 & $\begin{array}{c}\text { Between } \\
25 \text { and } 75 . \\
\text { (Adult, } \\
\text { Older } \\
\text { Adult) } \\
\text { Average: } \\
59.1\end{array}$ & $\begin{array}{c}58.6 \pm \\
8.5\end{array}$ & $\begin{array}{c}59.5 \pm \\
8.3\end{array}$ & 12 & $\begin{array}{l}\text { Seattle, } \\
\text { USA }\end{array}$ & $\begin{array}{l}622,927 \text { in } \\
2006\end{array}$ & $\begin{array}{l}10 \text { medical } \\
\text { centers within } \\
\text { Group Health } \\
\text { Research } \\
\text { Institute } \\
\text { (Not } \\
\text { underserved) }\end{array}$ & $\begin{array}{c}\text { Home BP } \\
\text { monitors, } \\
\text { instruction on } \\
\text { their use, and } \\
\text { proficiency } \\
\text { training on } \\
\text { web-based } \\
\text { communica- } \\
\text { tion }\end{array}$ & $\begin{array}{c}\text { Report once } \\
\text { every two } \\
\text { weeks (mea- } \\
\text { surement at } \\
\text { least twice a } \\
\text { week) }\end{array}$ & $\begin{array}{c}\text { Office SBP } \\
\text { and DBP } \\
\text { changes and } \\
\text { control of BP }\end{array}$ \\
\hline $\begin{array}{l}\text { Madsen } \\
\text { (2008) [47] }\end{array}$ & $\begin{array}{c}\text { Treated or } \\
\text { untreated } \\
\text { hypertensive } \\
\text { patients }\end{array}$ & 123 & 113 & $\begin{array}{c}\text { Between } \\
20 \text { and } 80 . \\
\text { Average } \\
\text { Age: } 55.9\end{array}$ & $\begin{array}{c}56.7 \pm \\
11.6\end{array}$ & $\begin{array}{c}55.0 \pm \\
11.7\end{array}$ & 6 & $\begin{array}{l}\text { Holstebro } \\
\text { (Den- } \\
\text { mark) }\end{array}$ & $\begin{array}{c}29,888 \text { in } \\
2004\end{array}$ & $\begin{array}{c}\text { Holstebro } \\
\text { Hospital } \\
\text { (Not } \\
\text { underserved) }\end{array}$ & $\begin{array}{c}\text { PDA- } \\
\text { embedded } \\
\text { mobile-web } \\
\text { phone } \\
\text { (mobile) }\end{array}$ & $\begin{array}{l}\text { Three times } \\
\text { a week } \\
\text { during the } \\
\text { first } 3 \\
\text { months and } \\
\text { once a week } \\
\text { during the } \\
\text { last } 3 \\
\text { months }\end{array}$ & $\begin{array}{c}\text { Difference in } \\
\text { systolic } \\
\text { daytime } \\
\text { ABPM } \\
\text { change }\end{array}$ \\
\hline $\begin{array}{c}\text { Parati } \\
(2009)[48]\end{array}$ & $\begin{array}{l}\text { Uncontrolled } \\
\text { hypertensive } \\
\text { patients }\end{array}$ & 111 & 187 & $\begin{array}{c}\text { Between } \\
17 \text { and } 75 . \\
\text { Average } \\
\text { age: } 57.5\end{array}$ & $\begin{array}{c}58.1 \pm \\
10.8\end{array}$ & $\begin{array}{c}57.2 \pm \\
10.7\end{array}$ & 6 & $\begin{array}{l}\text { Milan } \\
\text { (Italy) }\end{array}$ & $\begin{array}{c}1,198,182 \\
\text { in } 2006\end{array}$ & $\begin{array}{c}\text { Primary care } \\
\text { units in Milan } \\
\text { (Not } \\
\text { underserved) }\end{array}$ & $\begin{array}{l}\text { Telephone- } \\
\text { linked } \\
\text { computer } \\
\text { system }\end{array}$ & Regularly & $\begin{array}{l}\text { Percentage } \\
\text { of patients } \\
\text { who reached } \\
\text { normaliza- } \\
\text { tion of } \\
\text { BP }\end{array}$ \\
\hline $\begin{array}{c}\text { Park (2009) } \\
{[49]}\end{array}$ & $\begin{array}{c}\text { Obese } \\
\text { hypertensive } \\
\text { patients }\end{array}$ & 21 & 28 & $\begin{array}{l}\text { Average } \\
\text { age: } 53.8\end{array}$ & $\begin{array}{c}54.6 \pm \\
11.0\end{array}$ & $\begin{array}{c}53.2 \pm \\
6.9\end{array}$ & 2 & $\begin{array}{c}\text { Seoul } \\
\text { (S. Korea) }\end{array}$ & $\begin{array}{c}9,828,102 \\
\text { in } 2007\end{array}$ & $\begin{array}{l}\text { University- } \\
\text { affiliated } \\
\text { tertiary care } \\
\text { hospital } \\
\text { (Not } \\
\text { underserved) }\end{array}$ & $\begin{array}{l}\text { Telephone and } \\
\text { internet } \\
\text { transmission }\end{array}$ & Once a week & $\begin{array}{c}\text { Change in } \\
\text { blood } \\
\text { pressure, } \\
\text { body weight, } \\
\text { waist cir- } \\
\text { cumference, } \\
\text { and serum } \\
\text { lipid profile }\end{array}$ \\
\hline
\end{tabular}


Table 1. Cont.

\begin{tabular}{|c|c|c|c|c|c|c|c|c|c|c|c|c|c|}
\hline \multirow{2}{*}{ Study } & \multirow{2}{*}{$\begin{array}{c}\text { Included } \\
\text { Participants }\end{array}$} & \multicolumn{2}{|c|}{$\begin{array}{l}\text { Participants } \\
\text { Number }\end{array}$} & \multicolumn{3}{|c|}{$\begin{array}{l}\text { Participants' Age } \\
\text { Interval (Years) }\end{array}$} & \multirow{2}{*}{$\begin{array}{l}\text { Duration } \\
\text { (Months) }\end{array}$} & \multirow{2}{*}{$\begin{array}{c}\text { City } \\
\text { Name } \\
\text { (Country) }\end{array}$} & \multirow{2}{*}{$\begin{array}{l}\text { Population } \\
\text { of City }\end{array}$} & \multirow{2}{*}{ Setting } & \multirow{2}{*}{$\begin{array}{c}\text { Description } \\
\text { of } \\
\text { Intervention }\end{array}$} & \multirow{2}{*}{$\begin{array}{l}\text { Intervention } \\
\text { Frequency }\end{array}$} & \multirow{2}{*}{ Outcomes } \\
\hline & & UC & RBPM & $\begin{array}{c}\text { Age } \\
\text { Interval }\end{array}$ & UC & RBPM & & & & & & & \\
\hline $\begin{array}{c}\text { Varis } \\
\text { (2010) [50] }\end{array}$ & $\begin{array}{l}\text { Untreated } \\
\text { hypertensive } \\
\text { patients }\end{array}$ & 68 & 89 & $\begin{array}{l}\text { Between } \\
40 \text { and } 80\end{array}$ & $\begin{array}{l}\text { Not re- } \\
\text { ported }\end{array}$ & $\begin{array}{l}\text { Not re- } \\
\text { ported }\end{array}$ & 13 & $\begin{array}{l}\text { Helsinki } \\
\text { and } \\
\text { Tampere } \\
\text { and Turku } \\
\text { (Finland) }\end{array}$ & $\begin{array}{c}536,160 \\
\text { and } \\
194,594 \\
\text { and } \\
168,920 \text { In } \\
2007\end{array}$ & $\begin{array}{c}\text { Not } \\
\text { underserved }\end{array}$ & $\begin{array}{l}\text { Letter to } \\
\text { physician }\end{array}$ & $\begin{array}{l}\text { Every five } \\
\text { weeks (mea- } \\
\text { surement } \\
\text { every day) }\end{array}$ & $\begin{array}{c}\text { Changes in } \\
\mathrm{BP} \text { and } \\
\text { target } \mathrm{BP}\end{array}$ \\
\hline $\begin{array}{l}\text { Hoffmann- } \\
\text { Petersen } \\
\text { (2017) [51] }\end{array}$ & $\begin{array}{l}\text { Treated un- } \\
\text { complicated } \\
\text { hypertensive } \\
\text { patients }\end{array}$ & 181 & 175 & $\begin{array}{c}\text { Between } \\
55 \text { and } 64 \\
\text { Average: } \\
60.4\end{array}$ & $\begin{array}{c}60.4 \pm \\
2.9\end{array}$ & $\begin{array}{c}60.5 \pm \\
2.6\end{array}$ & 3 & $\begin{array}{l}\text { Holstebro } \\
\text { (Den- } \\
\text { mark) }\end{array}$ & $\begin{array}{c}30,885 \text { in } \\
2011\end{array}$ & $\begin{array}{c}\text { Holstebro } \\
\text { Regional } \\
\text { Hospital } \\
\text { (Not } \\
\text { underserved) }\end{array}$ & $\begin{array}{l}\text { Telephone and } \\
\text { e-mail com- } \\
\text { munication } \\
\text { (Telephone- } \\
\text { linked } \\
\text { computer } \\
\text { system) }\end{array}$ & $\begin{array}{l}\text { Once every } \\
\text { two weeks }\end{array}$ & $\begin{array}{l}\text { Daytime } \\
\text { ABPM } \\
\text { reduction } \\
\text { and } \\
\text { percentage } \\
\text { of target BP }\end{array}$ \\
\hline $\begin{array}{c}\text { Ionov } \\
\text { (2020) [52] }\end{array}$ & $\begin{array}{l}\text { Uncontrolled } \\
\text { hypertension } \\
\text { patients }\end{array}$ & 80 & 160 & $\begin{array}{c}\text { Between } \\
18 \text { and } 78\end{array}$ & $\begin{array}{l}49(20 \\
\text { to } 77)\end{array}$ & $\begin{array}{l}47(18 \\
\text { to } 78)\end{array}$ & 3 & $\begin{array}{c}\text { Saint- } \\
\text { Petersburg, } \\
\text { (Russia) }\end{array}$ & $\begin{array}{c}5,076,520 \\
\text { in } 2019\end{array}$ & $\begin{array}{c}\text { Federal } \\
\text { Medical } \\
\text { Research } \\
\text { Center } \\
\text { Hospital } \\
\text { (Not } \\
\text { underserved) }\end{array}$ & $\begin{array}{l}\text { Mobile phone } \\
\text { communica- } \\
\text { tion }\end{array}$ & $\begin{array}{c}\text { Once a week } \\
\text { (Measure- } \\
\text { ment: twice } \\
\text { a day) }\end{array}$ & $\begin{array}{c}\text { Change of } \\
\text { SBP and rate } \\
\text { of BP } \\
\text { control. }\end{array}$ \\
\hline $\begin{array}{c}\text { Logan } \\
\text { (2012) [53] }\end{array}$ & $\begin{array}{c}\text { Uncontrolled } \\
\text { hypertensive } \\
\text { and diabetic } \\
\text { patients }\end{array}$ & 51 & 54 & $\begin{array}{c}30 \text { or more } \\
\text { Average: } \\
62.9\end{array}$ & $\begin{array}{c}62.7 \pm \\
7.8\end{array}$ & $\begin{array}{c}63.1 \pm \\
9.0\end{array}$ & 12 & $\begin{array}{l}\text { Toronto } \\
\text { (Canada) }\end{array}$ & $\begin{array}{c}2,423,221 \\
\text { in } 2011\end{array}$ & $\begin{array}{c}\text { Mount Sinai } \\
\text { Hospital } \\
\text { (Not } \\
\text { underserved) }\end{array}$ & $\begin{array}{c}\text { Bluetooth- } \\
\text { enabled BP } \\
\text { device paired } \\
\text { with } \\
\text { smartphone } \\
\text { (mobile-web) }\end{array}$ & Twice a day & $\begin{array}{c}\text { Changes in } \\
\text { ambulatory } \\
\text { BP }\end{array}$ \\
\hline $\begin{array}{l}\text { Neumann } \\
\text { (2011) [54] }\end{array}$ & $\begin{array}{l}\text { Inadequately } \\
\text { treated } \\
\text { hypertensive } \\
\text { patients }\end{array}$ & 29 & 28 & $\begin{array}{l}\text { Between } \\
18 \text { and } 80 . \\
\text { Average } \\
\text { age: } 55.5\end{array}$ & $\begin{array}{c}56.2 \pm \\
17.4\end{array}$ & $\begin{array}{c}54.7 \pm \\
17.9\end{array}$ & 3 & $\begin{array}{l}\text { Göttingen } \\
\text { (Germany) }\end{array}$ & $\begin{array}{l}119,161 \text { in } \\
2009\end{array}$ & $\begin{array}{c}\text { Not } \\
\text { underserved }\end{array}$ & $\begin{array}{c}\text { Mobile } \\
\text { phone-linked } \\
\text { computer } \\
\text { system }\end{array}$ & Once a Day & BP Control \\
\hline
\end{tabular}


Table 1. Cont.

\begin{tabular}{|c|c|c|c|c|c|c|c|c|c|c|c|c|c|}
\hline \multirow{2}{*}{ Study } & \multirow{2}{*}{$\begin{array}{c}\text { Included } \\
\text { Participants }\end{array}$} & \multicolumn{2}{|c|}{$\begin{array}{l}\text { Participants } \\
\text { Number }\end{array}$} & \multicolumn{3}{|c|}{$\begin{array}{l}\text { Participants' Age } \\
\text { Interval (Years) }\end{array}$} & \multirow{2}{*}{$\begin{array}{l}\text { Duration } \\
\text { (Months) }\end{array}$} & \multirow{2}{*}{$\begin{array}{c}\text { City } \\
\text { Name } \\
\text { (Country) }\end{array}$} & \multirow{2}{*}{$\begin{array}{c}\text { Population } \\
\text { of City }\end{array}$} & \multirow{2}{*}{ Setting } & \multirow{2}{*}{$\begin{array}{c}\text { Description } \\
\text { of } \\
\text { Intervention }\end{array}$} & \multirow{2}{*}{$\begin{array}{l}\text { Intervention } \\
\text { Frequency }\end{array}$} & \multirow{2}{*}{ Outcomes } \\
\hline & & UC & RBPM & $\begin{array}{c}\text { Age } \\
\text { Interval }\end{array}$ & UC & RBPM & & & & & & & \\
\hline $\begin{array}{l}\text { Wakefield } \\
\text { (2011) [55] }\end{array}$ & $\begin{array}{c}\text { Type } 2 \\
\text { diabetics and } \\
\text { hypertensive } \\
\text { patients }\end{array}$ & 97 & 83 & $\begin{array}{c}\text { Between } \\
40 \text { and } 89 . \\
\text { Average: } \\
48.1\end{array}$ & $\begin{array}{c}67.9 \pm \\
9.9\end{array}$ & $\begin{array}{c}68.4 \pm \\
9.5\end{array}$ & 6 & $\begin{array}{l}\text { Iowa City } \\
\text { (USA) }\end{array}$ & $\begin{array}{l}67,548 \text { in } \\
2006\end{array}$ & $\begin{array}{l}\text { Iowa City VA } \\
\text { Health Care } \\
\text { System } \\
\text { (Not } \\
\text { underserved) }\end{array}$ & $\begin{array}{l}\text { Telephonic } \\
\text { transmission }\end{array}$ & Every day & $\begin{array}{l}\text { Changes in } \\
\text { hemoglobin } \\
A_{1 c} \text { and SBP }\end{array}$ \\
\hline $\begin{array}{l}\text { Bosworth } \\
\text { (2011) [56] }\end{array}$ & $\begin{array}{c}\text { Treated } \\
\text { hypertensive } \\
\text { patients }\end{array}$ & 137 & 127 & $\begin{array}{c}\text { Child, } \\
\text { Adult, } \\
\text { Older } \\
\text { Adult } \\
\text { Average } \\
\text { Age: } 63.5\end{array}$ & $\begin{array}{c}64 \pm \\
10\end{array}$ & $\begin{array}{c}63 \pm \\
11\end{array}$ & 12 & $\begin{array}{l}\text { Durham } \\
\text { (USA) }\end{array}$ & $\begin{array}{l}234,477 \text { in } \\
2006\end{array}$ & $\begin{array}{c}\text { Durham VA } \\
\text { Medical } \\
\text { Center } \\
\text { (Not } \\
\text { underserved) }\end{array}$ & $\begin{array}{l}\text { Telephonic } \\
\text { transmission }\end{array}$ & Once a day & $\begin{array}{l}\text { 1. BP control } \\
\text { 2. SBP and } \\
\text { DBP change }\end{array}$ \\
\hline $\begin{array}{l}\text { Migneault } \\
\text { (2012) [57] }\end{array}$ & $\begin{array}{c}\text { African } \\
\text { American } \\
\text { hypertensive } \\
\text { patients }\end{array}$ & 140 & 125 & $\begin{array}{l}35 \text { or more. } \\
\text { Average } \\
\text { age: } 56.5\end{array}$ & $\begin{array}{c}56.8 \pm \\
11.4\end{array}$ & $\begin{array}{c}56.3 \pm \\
10.6\end{array}$ & 8 & $\begin{array}{l}\text { Boston } \\
\text { (USA) }\end{array}$ & $\begin{array}{l}590,971 \text {, in } \\
2003\end{array}$ & $\begin{array}{c}\text { Boston } \\
\text { Medical } \\
\text { Center } \\
\text { primary care } \\
\text { practices of a } \\
\text { large, } \\
\text { safety-net } \\
\text { hospital and } \\
\text { four affiliated } \\
\text { community } \\
\text { health centers. } \\
\text { (Underserved) }\end{array}$ & $\begin{array}{l}\text { Automated, } \\
\text { computer- } \\
\text { based, } \\
\text { interactive } \\
\text { telephone } \\
\text { counseling } \\
\text { system }\end{array}$ & Once a week & $\begin{array}{l}\text { Change in } \\
\text { diet quality, } \\
\text { leisure time } \\
\text { physical } \\
\text { activity of } \\
\text { moderate- } \\
\text { or-greater } \\
\text { intensity, } \\
\text { and } \\
\text { adherence to } \\
\text { the antihy- } \\
\text { pertensive } \\
\text { medication } \\
\text { regimen and } \\
\text { change in } \\
\text { BP. }\end{array}$ \\
\hline
\end{tabular}


Table 1. Cont.

\begin{tabular}{|c|c|c|c|c|c|c|c|c|c|c|c|c|c|}
\hline \multirow{2}{*}{ Study } & \multirow{2}{*}{$\begin{array}{c}\text { Included } \\
\text { Participants }\end{array}$} & \multicolumn{2}{|c|}{$\begin{array}{c}\text { Participants } \\
\text { Number }\end{array}$} & \multicolumn{3}{|c|}{$\begin{array}{c}\text { Participants' Age } \\
\text { Interval (Years) }\end{array}$} & \multirow{2}{*}{$\begin{array}{l}\text { Duration } \\
\text { (Months) }\end{array}$} & \multirow{2}{*}{$\begin{array}{c}\text { City } \\
\text { Name } \\
\text { (Country) }\end{array}$} & \multirow{2}{*}{$\begin{array}{l}\text { Population } \\
\text { of City }\end{array}$} & \multirow{2}{*}{ Setting } & \multirow{2}{*}{$\begin{array}{l}\text { Description } \\
\text { of } \\
\text { Intervention }\end{array}$} & \multirow{2}{*}{$\begin{array}{l}\text { Intervention } \\
\text { Frequency }\end{array}$} & \multirow{2}{*}{ Outcomes } \\
\hline & & UC & RBPM & $\begin{array}{c}\text { Age } \\
\text { Interval }\end{array}$ & UC & RBPM & & & & & & & \\
\hline $\begin{array}{l}\text { Park (2012) } \\
{[58]}\end{array}$ & $\begin{array}{c}\text { Post- } \\
\text { menopausal } \\
\text { obese } \\
\text { hypertensive } \\
\text { patients }\end{array}$ & 33 & 34 & $\begin{array}{l}\text { Average } \\
\text { age: } 56.7\end{array}$ & $\begin{array}{l}57.6 \pm \\
5.5\end{array}$ & $\begin{array}{c}55.8 \pm \\
5.7\end{array}$ & 3 & $\begin{array}{c}\text { Seoul } \\
\text { (S. Korea) }\end{array}$ & $\begin{array}{c}9,828,102 \\
\text { in } 2007\end{array}$ & $\begin{array}{c}\text { University } \\
\text { medical center } \\
\text { (Not } \\
\text { underserved) }\end{array}$ & $\begin{array}{l}\text { Reporting on } \\
\text { website. } \\
\text { Mobile and } \\
\text { internet } \\
\text { transmission }\end{array}$ & Once a week. & $\begin{array}{l}\text { Change in } \\
\text { waist cir- } \\
\text { cumference, } \\
\text { body weight, } \\
\text { and blood } \\
\text { pressure, } \\
\text { fasting } \\
\text { plasma } \\
\text { glucose, and } \\
\text { serum lipid } \\
\text { levels }\end{array}$ \\
\hline $\begin{array}{c}\text { Bove } \\
\text { (2013) [59] }\end{array}$ & $\begin{array}{c}\text { Systolic } \\
\text { hypertensive } \\
\text { patients }\end{array}$ & 107 & 99 & $\begin{array}{c}\text { Between } \\
18 \text { and } 85 \\
\text { (Adult, } \\
\text { Older } \\
\text { Adult) } \\
\text { Average: } \\
59.6\end{array}$ & $\begin{array}{c}58.2 \pm \\
13.5\end{array}$ & $\begin{array}{c}61.0 \pm \\
13.6\end{array}$ & 6 & $\begin{array}{l}\text { Philadelphia } \\
\text { Wilmington, } \\
\text { USA }\end{array}$ & $\begin{array}{l}\text { / 1,480,457/ } \\
109,499 \text { in } \\
2010\end{array}$ & $\begin{array}{l}\text { University } \\
\text { hospital (Un- } \\
\text { derserved) }\end{array}$ & $\begin{array}{l}\text { Telephone and } \\
\text { internet-based } \\
\text { System }\end{array}$ & Once a day & $\begin{array}{l}\text { BP control at } \\
6 \text { months }\end{array}$ \\
\hline $\begin{array}{l}\text { Wakefield } \\
\text { (2014) [60] }\end{array}$ & $\begin{array}{c}\text { Type } 2 \\
\text { diabetics and } \\
\text { uncontrolled } \\
\text { hypertensive } \\
\text { patients }\end{array}$ & 43 & 40 & $\begin{array}{l}18 \text { or more. } \\
\text { Average: } \\
60.0\end{array}$ & $\begin{array}{c}62.5 \pm \\
10.9\end{array}$ & $\begin{array}{c}57.7 \pm \\
10.8\end{array}$ & 3 & $\begin{array}{l}\text { Columbia } \\
\text { (USA) }\end{array}$ & $\begin{array}{l}112,498 \text { in } \\
2010\end{array}$ & $\begin{array}{l}\text { University } \\
\text { hospital } \\
\text { (Not } \\
\text { underserved) }\end{array}$ & $\begin{array}{l}\text { Web System } \\
\text { through } \\
\text { mobile phone } \\
\text { or personal } \\
\text { computer }\end{array}$ & $\begin{array}{c}\text { Twice a } \\
\text { week (Mea- } \\
\text { surement: } \\
\text { every day) }\end{array}$ & $\begin{array}{l}\text { Changes in } \\
\text { hemoglobin } \\
\mathrm{A}_{1 \mathrm{c}} \text { and SBP }\end{array}$ \\
\hline
\end{tabular}


Table 1. Cont.

\begin{tabular}{|c|c|c|c|c|c|c|c|c|c|c|c|c|c|}
\hline \multirow{2}{*}{ Study } & \multirow{2}{*}{$\begin{array}{c}\text { Included } \\
\text { Participants }\end{array}$} & \multicolumn{2}{|c|}{$\begin{array}{l}\text { Participants } \\
\text { Number }\end{array}$} & \multicolumn{3}{|c|}{$\begin{array}{l}\text { Participants' Age } \\
\text { Interval (Years) }\end{array}$} & \multirow{2}{*}{$\begin{array}{l}\text { Duration } \\
\text { (Months) }\end{array}$} & \multirow{2}{*}{$\begin{array}{c}\text { City } \\
\text { Name } \\
\text { (Country) }\end{array}$} & \multirow{2}{*}{$\begin{array}{l}\text { Population } \\
\text { of City }\end{array}$} & \multirow{2}{*}{ Setting } & \multirow{2}{*}{$\begin{array}{c}\text { Description } \\
\text { of } \\
\text { Intervention }\end{array}$} & \multirow{2}{*}{$\begin{array}{l}\text { Intervention } \\
\text { Frequency }\end{array}$} & \multirow{2}{*}{ Outcomes } \\
\hline & & UC & RBPM & $\begin{array}{c}\text { Age } \\
\text { Interval }\end{array}$ & UC & RBPM & & & & & & & \\
\hline $\begin{array}{l}\text { Yi (2015) } \\
\text { [61] }\end{array}$ & $\begin{array}{c}\text { Uncontrolled } \\
\text { hypertensive } \\
\text { patients }\end{array}$ & 332 & 329 & $\begin{array}{c}18 \text { or more. } \\
\text { Average: } \\
61.3\end{array}$ & $\begin{array}{c}61.3 \pm \\
12.2\end{array}$ & $\begin{array}{c}61.3 \pm \\
11.9\end{array}$ & 9 & $\begin{array}{c}\text { Bronx and } \\
\text { Brooklyn } \\
\text { and New } \\
\text { York } \\
\text { (USA) }\end{array}$ & $\begin{array}{c}1,308,242 \\
\text { and } \\
2,172,989 \\
\text { and } \\
7,721,458 \\
\text { in } 2010\end{array}$ & $\begin{array}{c}\text { Riverdale } \\
\text { Family } \\
\text { Practice } \\
\text { (Bronx), } \\
\text { Lutheran } \\
\text { Family Health } \\
\text { Centers } \\
\text { (Brooklyn), } \\
\text { New York City } \\
\text { Department of } \\
\text { Health and } \\
\text { Mental } \\
\text { Hygiene (New } \\
\text { York City), } \\
\text { Heritage } \\
\text { Health Care } \\
\text { (New York } \\
\text { City) } \\
\text { (Underserved) }\end{array}$ & $\begin{array}{l}\text { Telephone- } \\
\text { linked } \\
\text { computer } \\
\text { system }\end{array}$ & $\begin{array}{c}\text { Once a } \\
\text { month (Mea- } \\
\text { surement: } \\
\text { every day) }\end{array}$ & $\begin{array}{c}\text { Change in } \\
\text { SBP and } \\
\text { DBP and } \\
\text { achieve- } \\
\text { ment of BF } \\
\text { control }\end{array}$ \\
\hline
\end{tabular}

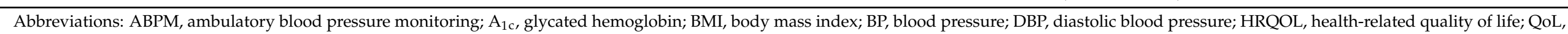
quality of life; RBPM, remote blood pressure monitoring; SBP, systolic blood pressure; SMS, short message service; UC, usual care. 


\subsection{Risk Assessment}

To check for bias in RCT studies, the Cochrane Group's RoB tool of the Cochrane group was used for domain analysis based on a checklist. Across seven domains, a low risk of selection bias related to sequence generation or allocation concealment was shown. Similarly, the risk of detection bias related to blinding of personnel and patients was appropriately reported. Concerning attrition bias (incomplete outcome data), an unclear or sufficiently high risk was shown that raised concern in a number of studies; however, as most studies showed a low risk $(\geq 4)$ across the seven domains, the overall RoB was deemed to be low [62].

Egger's regression intercept was $4.516(1.363-7.669 ; p=0.005)$ in two-tailed 95\% CIs [37]. The number of studies needed to attain $p>0.05$ for a classic fail-safe $\mathrm{N}$ was 5085 . The point estimate of SBP in Duval and Tweedie's trim-and-fill analysis (SMD, $0.507 \mathrm{mmHg}$ $(0.339-0.645, p<0.001)$; WMD $4.464 \mathrm{mmHg}(p<0.001))$ coincided with the summary effect size, while no imputed study was found in the funnel plot (Figure 2) [63]. The SMD of DBP was $0.253(0.215-0.292)$, and no study was trimmed (Figure 3$)$. In the analysis of the rate of target BP achievement, RR was 1.237 (1.107-1.381), three studies were imputed, and the adjusted value was 1.161 (1.032-1.306, Figure 4). Although RoB assessment detected a certain level of publication bias, the overall data were statistically significant and the analysis results were not rejected.

Funnel Plot of Standard Error by Std diff in means

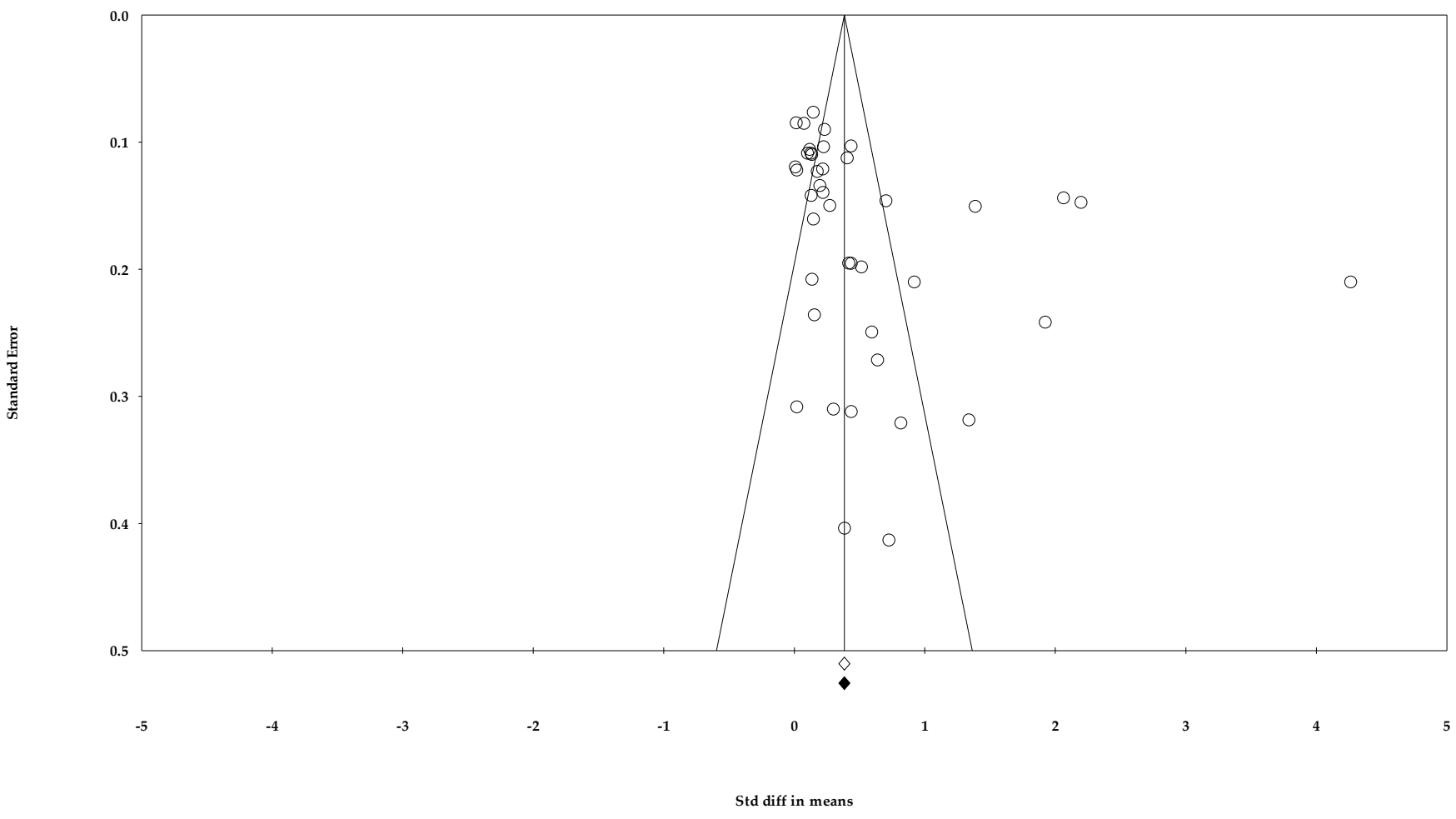

Figure 2. A funnel plot of the standardized mean difference in systolic blood pressure. Note: summary effect size $(\diamond)$, summary effect size of imputed studies $(\bullet)$, individual study $(\bigcirc)$. 
Funnel Plot of Standard Error by Std diff in means

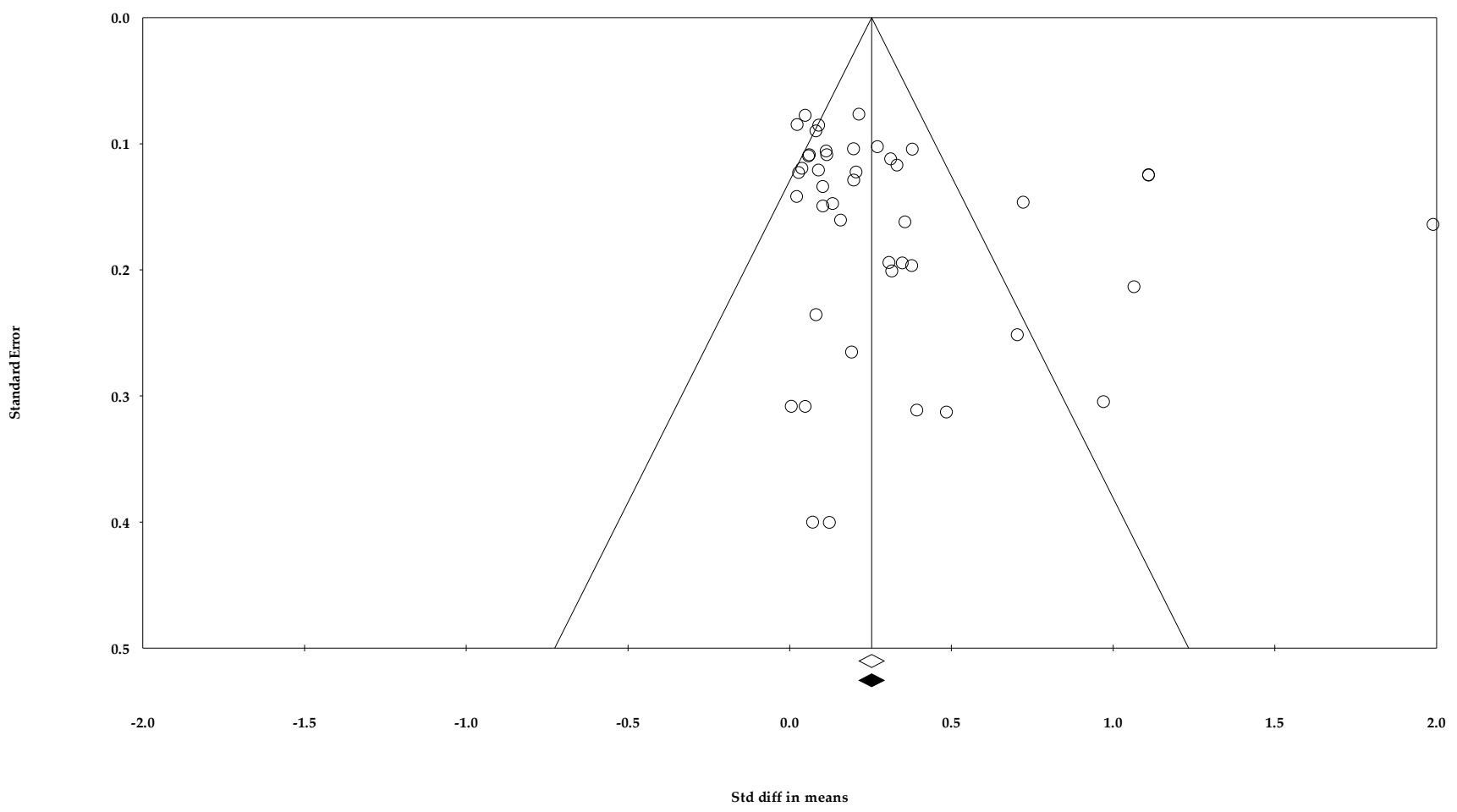

Figure 3. A funnel plot of standardized mean difference in diastolic blood pressure. Note: summary effect size $(\diamond)$, summary effect size of imputed studies $(\bullet)$, individual study $(\bigcirc)$.

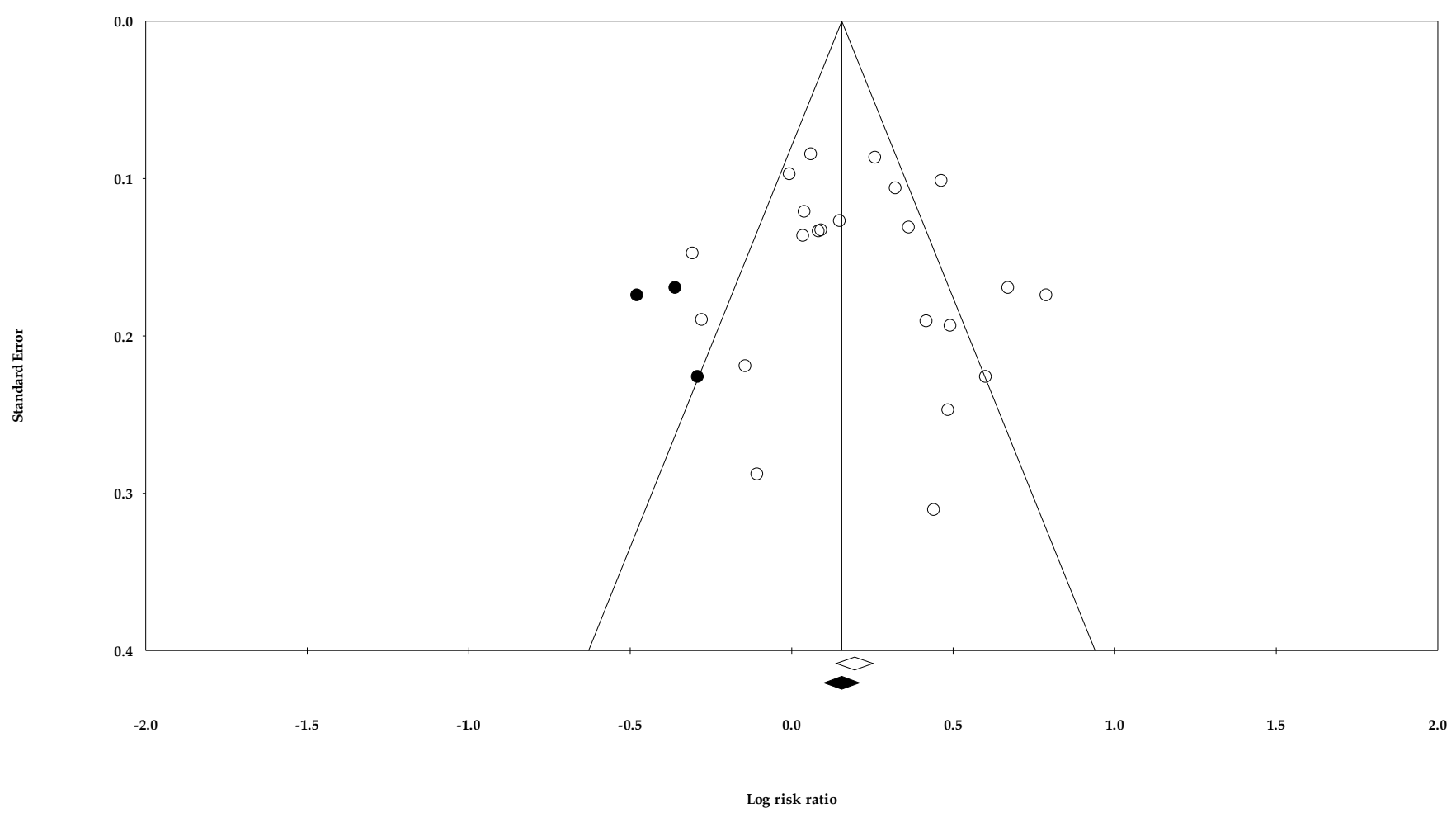

Figure 4. A funnel plot of relative risk for the target blood pressure rate. Note: summary effect size $(\diamond)$, imputed study $(\bullet)$, summary effect size of imputed studies $(\bullet)$, individual study $(\bigcirc)$. 
A sensitivity test was performed for studies that had been included to prevent smallstudy effects, excluding those with a sample size of $\leq 50$ for the RBPM group [64]. The test results showed an SMD of $0.501 \mathrm{mmHg}(0.313-0.689, p<0.001)$ and a WMD of $4.238 \mathrm{mmHg}$ $(p<0.001)$, indicating that the difference from the overall summary effect size was not clinically significant and that the potential small-study effect was not significant in this study.

\subsection{Primary Outcomes}

\subsubsection{Systolic Blood Pressure}

Across 32 independent studies (48 comparisons), 11,395 patients (UC group, $n=$ 5666; RBPM group, $n=5,729)$ were analyzed for SBP [25-35,41-61]. The summary SMD was $0.507(0.339-0.675, p<0.001)$, showing an above moderate effect size, and the WMD after conversion was $4.464 \mathrm{mmHg}(3.371-5.556, p<0.001$; Figure 5). The between-group heterogeneity was significant $\left(\mathrm{I}^{2}=70.908 \%, p<0.001\right)$. To determine the effect of individual studies on the total summary effect size, a sensitivity test was performed using the "one study removed" method, whereby each study was sequentially omitted (Appendix B). Here, the point estimate of the summary effect size showed no significant difference and no outliers were detected.

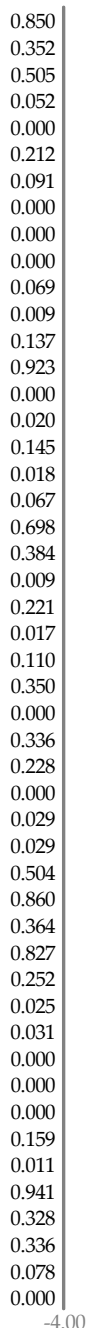

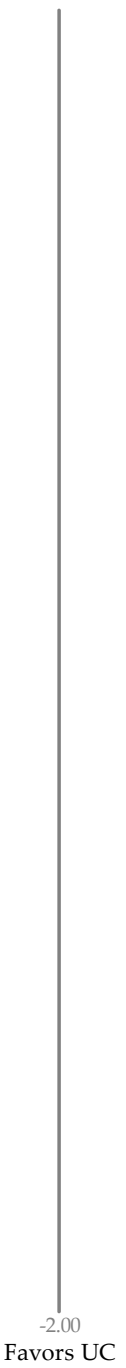

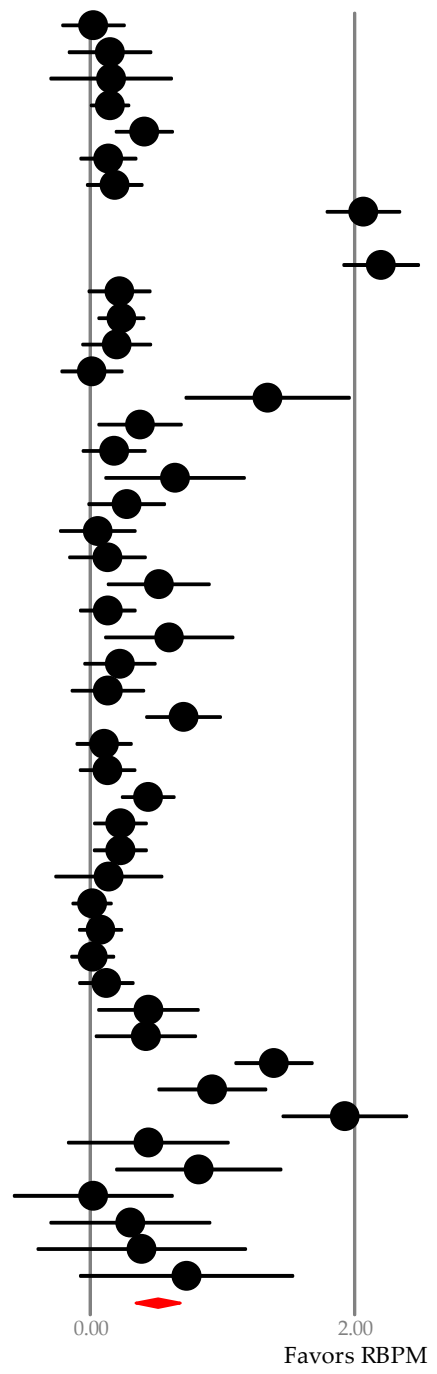

Figure 5. A forest plot of standardized mean difference in systolic blood pressure. Note: point estimate of individual study $(\bullet)$, summary effect size $(\bullet)$; SBP, systolic blood pressure; UC, usual care; RBPM, remote blood pressure monitoring. 
When the average effect of RBPM was chronologically divided into three timeframes and compared with the UC group (Phase I, inception of RBPM to 2000; phase II, 2001-2010; phase III, 2011-2020), the WMD was $1.515 \mathrm{mmHg}(n=2,-4.031-7.061, p=0.592$; $\left.\mathrm{I}^{2}=0.000 \%, p=0.478\right)$ in phase I $[41,42], 4.333 \mathrm{mmHg}(n=14,2.338-6.328, p<0.001$; $\left.\mathrm{I}^{2}=38.554, p<0.001\right)$ in phase II $[25,29,43-50]$, and $4.719 \mathrm{mmHg}(n=32,3.343-6.094$, $\left.p<0.001 ; \mathrm{I}^{2}=77.361 \%, p<0.001\right)$ in phase III [26-28,30-35,51-61].

\subsubsection{Diastolic Blood Pressure}

To determine the effect of RBPM on DBP, data concerning 10,482 patients (UC group, $n=5192$; RBPM group, $n=5290$ ) were analyzed across 29 studies (44 comparisons) [25,2735,41-54,56-59,61]. Compared with the UC group, the RBPM group showed greater BP reduction (SMD, $0.315 \mathrm{mmHg}(0.209-0.402), p<0.001$; WMD, $2.075 \mathrm{mmHg}(1.399-2.750)$ $p<0.001$ ) after conversion (Figure 6$)$. The between-study heterogeneity was substantial $\left(\mathrm{I}^{2}\right.$, $68.021 \% ; p<0.001)$. No outliers were detected in the sensitivity test performed through sequentially omitting each study.

\begin{tabular}{|c|c|c|c|c|c|c|}
\hline \multirow[t]{2}{*}{ Outcome } & \multirow[t]{2}{*}{ Author (year) (ref.) } & \multicolumn{5}{|c|}{ Statistics for each study } \\
\hline & & $\begin{array}{l}\text { Std diff } \\
\text { in means }\end{array}$ & $\begin{array}{c}\text { Lower } \\
\text { limit }\end{array}$ & $\begin{array}{c}\text { Upper } \\
\text { limit }\end{array}$ & Z-Value & $p$-Value \\
\hline DBP & Friedman,1996 & 0.207 & -0.034 & 0.447 & 1.684 & 0.092 \\
\hline DBP & Hill,1999 & 0.159 & -0.156 & 0.474 & 0.988 & 0.323 \\
\hline DBP & McMahon,2005 & 0.083 & -0.379 & 0.545 & 0.351 & 0.725 \\
\hline DBP & Shea,2006 & 0.215 & 0.065 & 0.366 & 2.801 & 0.005 \\
\hline DBP & Artinian,2007A & 0.314 & 0.094 & 0.534 & 2.793 & 0.005 \\
\hline DBP & Artinian,2007B & 0.060 & -0.156 & 0.275 & 0.542 & 0.588 \\
\hline DBP & Artinian,2007C & 0.062 & -0.152 & 0.276 & 0.569 & 0.569 \\
\hline DBP & Bosworth,2007A & 0.333 & 0.104 & 0.563 & 2.843 & 0.004 \\
\hline DBP & Bosworth,2007B & 1.111 & 0.866 & 1.356 & 8.896 & 0.000 \\
\hline DBP & Bosworth,2007C & 1.111 & 0.865 & 1.357 & 8.866 & 0.000 \\
\hline DBP & Carrasco,2008 & 0.090 & -0.147 & 0.328 & 0.745 & 0.456 \\
\hline DBP & Green,2008 & 0.083 & -0.094 & 0.259 & 0.916 & 0.359 \\
\hline DBP & Madsen,2008 & 0.104 & -0.159 & 0.367 & 0.774 & 0.439 \\
\hline DBP & Parati,2009 & 0.039 & -0.196 & 0.274 & 0.328 & 0.743 \\
\hline DBP & Park MJ,2009 & 0.972 & 0.374 & 1.569 & 3.187 & 0.001 \\
\hline DBP & Varis,2010 & 0.358 & 0.040 & 0.676 & 2.204 & 0.027 \\
\hline DBP & Bosworth,2011 & 0.029 & -0.212 & 0.270 & 0.235 & 0.814 \\
\hline DBP & Neumann,2011 & 0.193 & -0.327 & 0.714 & 0.727 & 0.467 \\
\hline DBP & Hebert,2012A & 0.134 & -0.155 & 0.424 & 0.908 & 0.364 \\
\hline DBP & Hebert,2012B & 0.104 & -0.189 & 0.398 & 0.696 & 0.487 \\
\hline DBP & Logan,2012 & 0.379 & -0.007 & 0.765 & 1.923 & 0.054 \\
\hline DBP & Migneault,2012 & 0.116 & -0.098 & 0.330 & 1.065 & 0.287 \\
\hline DBP & Park MJ, 2012 & 0.705 & 0.212 & 1.199 & 2.800 & 0.005 \\
\hline DBP & Bove,2013 & 0.200 & -0.054 & 0.453 & 1.545 & 0.122 \\
\hline DBP & Cicolini,2013A & 0.023 & -0.255 & 0.302 & 0.163 & 0.871 \\
\hline DBP & Cicolini,2013B & 0.723 & 0.436 & 1.011 & 4.931 & 0.000 \\
\hline DBP & Kim KB,2014A & 0.273 & 0.071 & 0.474 & 2.656 & 0.008 \\
\hline DBP & Kim KB,2014B & 0.381 & 0.176 & 0.586 & 3.638 & 0.000 \\
\hline DBP & Kim KB,2014C & 0.199 & -0.006 & 0.404 & 1.907 & 0.057 \\
\hline DBP & $Y i, 2015$ & 0.050 & -0.103 & 0.202 & 0.636 & 0.525 \\
\hline DBP & Rubinstein, 2016A & 0.091 & -0.076 & 0.259 & 1.068 & 0.285 \\
\hline DBP & Rubinstein, 2016B & 0.024 & -0.142 & 0.191 & 0.287 & 0.774 \\
\hline DBP & Hoffmann,2017 & 0.114 & -0.094 & 0.322 & 1.077 & 0.281 \\
\hline DBP & Pan,2018A & 0.350 & -0.032 & 0.732 & 1.794 & 0.073 \\
\hline DBP & Pan,2018B & 0.309 & -0.073 & 0.690 & 1.586 & 0.113 \\
\hline DBP & Ionov, 2020 & 1.991 & 1.669 & 2.313 & 12.113 & 0.000 \\
\hline DBP & Mohsen,2020A & 0.317 & -0.077 & 0.712 & 1.577 & 0.115 \\
\hline DBP & Mohsen,2020B & 1.066 & 0.647 & 1.485 & 4.987 & 0.000 \\
\hline DBP & Rahmani Pour,2020A & A 0.486 & -0.128 & 1.100 & 1.553 & 0.120 \\
\hline DBP & Rahmani Pour,2020B & 0.006 & -0.598 & 0.611 & 0.021 & 0.983 \\
\hline DBP & Rahmani Pour,2020C & 0.049 & -0.555 & 0.654 & 0.160 & 0.873 \\
\hline DBP & Rahmani Pour,2020D & 0.395 & -0.216 & 1.005 & 1.267 & 0.205 \\
\hline DBP & Zha,2020A & 0.124 & -0.661 & 0.909 & 0.310 & 0.757 \\
\hline DBP & Zha,2020B & 0.072 & -0.712 & 0.857 & 0.181 & 0.856 \\
\hline & & 0.315 & 0.209 & 0.422 & 5.801 & 0.000 \\
\hline
\end{tabular}

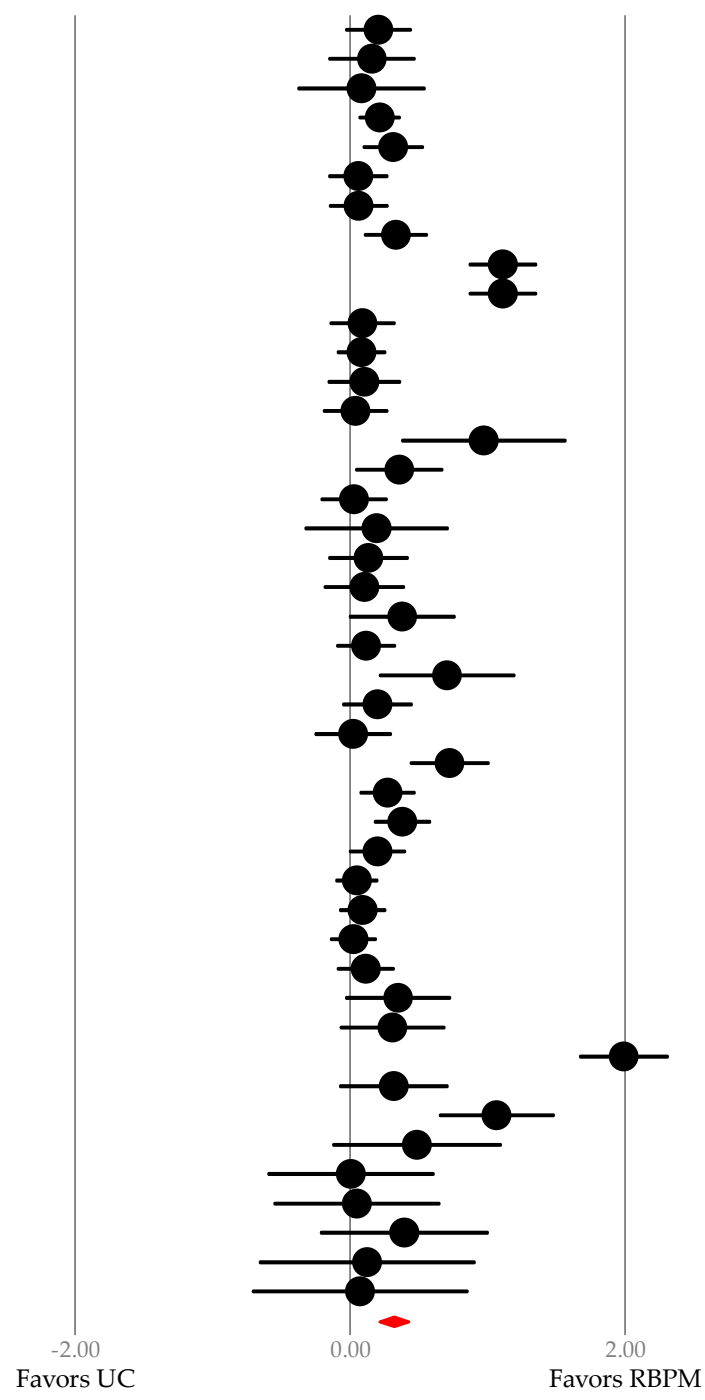

Figure 6. A forest plot of standardized mean difference in diastolic blood pressure. Note: point estimate of individual study $(\bullet)$, summary effect size $(\bullet)$; DBP, diastolic blood pressure; UC, usual care; RBPM, remote blood pressure monitoring. 
The WMD according to time interval was $2.059 \mathrm{mmHg}$ in phase I $(n=2,-1.143-5.262$, $\left.p=0.208 ; \mathrm{I}^{2}=0.000 \%, p=0.45\right)[41,42], 1.587 \mathrm{mmHg}$ in phase II $(n=14,0.421-2.753, p<0.001$; $\left.\mathrm{I}^{2}=17.407 \%, p<0.001\right)[25,29,43-50]$, and $2.348 \mathrm{mmHg}$ in phase III $(n=28 ; 1.480-3.216$, $\left.p<0.001 ; \mathrm{I}^{2}=76.230 \%, p<0.001\right)[26-28,30-35,51-61]$.

\subsubsection{Target Blood Pressure Rate}

To determine the effect of RBPM, the rate of BP control was estimated based on BP normalization criteria defined in each primary study. Across 16 studies (25 comparisons), the data of 2655 patients in the UC group and 2816 patients in the RBPM group were comprehensively analyzed $[13,25,30-33,45-47,50-53,59,61]$. Compared with the UC group, the RBPM group showed a significant effect, with an approximately $23.7 \%$ higher improvement in BP control based on $\mathrm{RR}(\mathrm{RR}=1.226(1.107-1.358), p<0.001$; Figure 7). The between-study heterogeneity was substantial $\left(\mathrm{I}^{2}=70.656 \% ; p<0.001\right)$. No significant difference in summary effect size was found in the sensitivity test.

\begin{tabular}{ll} 
Author, year & Duratio \\
\cline { 2 - 2 } & \\
& \\
Bosworth, 2007A6.000 \\
Bosworth, 2007B 6.000 \\
Bosworth, 2007C6.000 \\
Bove, 2013 & 6.000 \\
Carasco, 2008 & 6.000 \\
Cicolini, 2013A & 3.000 \\
Cicolini, 2013B & 6.000 \\
Green, 2008 & 12.000 \\
Hebert, 2011A & 9.000 \\
Hebert, 2011B & 9.000 \\
Hoffmann, 2017 & 3.000 \\
Ionov, 2020 & 3.000 \\
Kim KB, 2014A & 6.000 \\
Kim KB, 2014B & 12.000 \\
Logan, 2016 & 12.000 \\
Madsen, 2008 & 6.000 \\
Migneault, 2012 & 9.000 \\
Mohsen, 2020A & 3.000 \\
Mohsen, 2020B & 6.000 \\
Neumann, 2011 & 3.000 \\
Pan, 2018A & 3.000 \\
Pan, 2018B & 6.000 \\
Parati, 2009 & 6.000 \\
Varis, 2010 & 12.000 \\
Yi, 2015 & 12.000 \\
&
\end{tabular}

\begin{tabular}{|c|c|c|c|c|}
\hline \multirow[t]{2}{*}{ Dutcome } & \multicolumn{4}{|c|}{ Statistics for each study } \\
\hline & $\begin{array}{l}\text { Risk } \\
\text { ratio }\end{array}$ & $\begin{array}{c}\text { Lower } \\
\text { limit }\end{array}$ & $\begin{array}{c}\text { Upper } \\
\text { limit }\end{array}$ & p-Value \\
\hline Targeted BP & 1.036 & 0.793 & 1.353 & 0.795 \\
\hline Targeted BP & 1.096 & 0.845 & 1.422 & 0.491 \\
\hline Targeted BP & 1.087 & & 1.412 & 0.532 \\
\hline Targeted BP & 1.040 & 0.821 & .319 & 0.744 \\
\hline Targeted BP & 1.062 & 0.900 & 1.253 & 0.478 \\
\hline Targeted BP & 1.437 & 1.112 & 1.858 & 0.006 \\
\hline Targeted BP & 1.590 & 1.303 & 1.940 & 0.000 \\
\hline Targeted BP & 1.161 & 0.905 & 488 & 0.241 \\
\hline$d B P$ & 1.091 & 0.779 & 528 & 0.612 \\
\hline ed BP & 1.149 & & 519 & 0.428 \\
\hline Targeted $B P$ & 0.867 & & & 0.514 \\
\hline Targeted BP & 2.200 & 1.564 & 3.095 & 0.000 \\
\hline Cargeted BP & 1.379 & 1.120 & 1.698 & 0.002 \\
\hline Targeted BP & 1.295 & 1.093 & 1.535 & 0.003 \\
\hline Targeted BP & 1.624 & 1.000 & 2.636 & 0.050 \\
\hline Targeted BP & 0.757 & 0.522 & 1.098 & 0.143 \\
\hline Targeted BP & 1.243 & 0.503 & 3.071 & 0.638 \\
\hline Targeted BP & 1.824 & 1.171 & 2.840 & 0.008 \\
\hline Targeted BP & 1.955 & & 724 & 0.000 \\
\hline Targeted BP & 1.554 & & & 0.156 \\
\hline Targeted $\mathrm{BP}$ & 1.635 & 1.119 & 2.389 & 0.011 \\
\hline Targeted BP & 1.518 & 1.044 & 2.205 & 0.029 \\
\hline Targeted BP & 0.736 & 0.551 & 0.983 & 0.038 \\
\hline Targeted BP & 0.899 & 0.511 & 1.580 & 0.711 \\
\hline Targeted BP & 0.994 & 0.821 & 1.202 & 0.947 \\
\hline & 1.226 & 1.107 & 1.358 & 0.000 \\
\hline
\end{tabular}

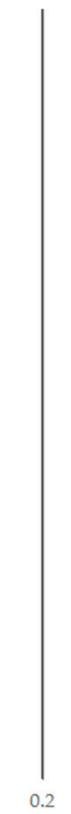

Favors UC

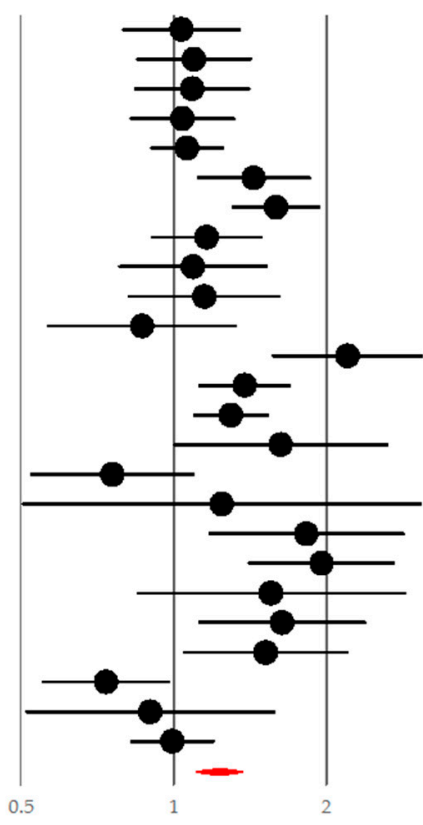

Favors RBPM

Figure 7. Risk ratio of target blood pressure using remote blood pressure monitoring. Note: point estimate of individual study $(\bullet)$, summary effect size $(\bullet)$; BP, blood pressure; UC, usual care; RBPM, remote blood pressure monitoring.

\subsection{Subgroup Analysis \\ 3.4.1. City Size}

Generally accepted international criteria define city size according to population size in a given area. In this study, a metropolitan city was defined as a city with a population of at least one million. Thus, the RCT studies included in this study were categorized based on city size as either a small-to-medium-sized city study or a large city study, and the two categories were analyzed separately. Population size was estimated from the data of the latest international population survey performed in the nearest period of time to this study. Of the 48 studies, 22 were conducted in small-to-medium cities [25,28,29,32,41-43,46,47,50, 51,54-57] and 26 were conducted in large cities [26,27,30,31,33-35,44,45,48,49,52,53,58-61]. For the former, the SBP showed a WMD of $3.860 \mathrm{mmHg}(2.271-5.450, p<0.001)$ without between-study heterogeneity $\left(\mathrm{I}^{2}=0.000, p=0.478\right.$; Tau $\left.{ }^{2}=0.000\right)$. For the latter, the SBP showed a WMD of $5.056 \mathrm{mmHg}(3.503-6.609, p<0.001)$ with a significant level of between- 
study heterogeneity $\left(\mathrm{I}^{2}=82.177 \%, p<0.001\right.$, $\left.\mathrm{Tau}^{2}=17.368\right)$; the magnitude of the effect size was above moderate.

\subsubsection{Medically Underserved Areas}

The presence of MUAs for each group was reflected in the analysis only if the study clearly indicated the respective area. As a result, 17 studies were categorized as MUAs [28, $29,31,32,35,41,44,57,59,61]$ and 31 as non-MUAs [25-27,30,33,34,42,43,45-56,58,60]. In terms of MUAs, the effect of RBPM on SBP showed a WMD of $3.213 \mathrm{mmHg}(1.521-4.905$, $p<0.001)$, with $\mathrm{I}^{2}=48.904 \%\left(p=0.012\right.$, Tau $\left.{ }^{2}=2.793\right)$, indicating a moderate degree of between-study heterogeneity based on Cohen's rule of thumb. In contrast, in non-MUAs, the effect of RBPM on SBP showed a WMD of $5.224 \mathrm{mmHg}(3.878-6.569 ; p<0.001)$, with $\mathrm{I}^{2}=73.152 \%\left(p<0.001, \mathrm{Tau}^{2}=12.943\right)$.

\subsubsection{Duration of Intervention}

The effect of reduced SBP based on the WMD varied according to the duration of the intervention. For an intervention duration $\leq 3$ months $[27-30,33,34,49,51,52,54,58,60]$, the effect was a WMD of $6.219 \mathrm{mmHg}\left(n=15,3.970-8.468, p<0.001 ; \mathrm{I}^{2}=70.060, p<0.001\right)$. For 6 months $[26-30,32,33,35,42,45,47,48,55,59]$, the effect was a WMD of $4.491 \mathrm{mmHg}$ $\left(n=14,2.461-6.521, p<0.001 ; \mathrm{I}^{2}=84.562, p<0.001\right)$. For 12 months, the effect was a WMD of $3.446 \mathrm{mmHg}\left(n=12,1.209-5.683, p=0.003 ; \mathrm{I}^{2}=34.656, p=0.113\right)$. The rate of BP control had an RR of $1.540(n=6,1.223-1.939, p<0.001)$ after 3 months $[27,30,33,51,52,54]$, an RR of $1.159(n=11,1.002-1.341, p=0.047)$ after 6 months $[25,27,30,32,33,45,47,48,59]$, and an RR of $1.167(n=5,0.930-1.464, p=0.183)$ after 12 months $[32,46,50,53,61]$ (Appendix D, Figure A3).

\subsubsection{Setting}

The BP reducing effect was analyzed according to the size of the medical institution where RBPM was mainly performed. In primary care clinics, the WMD was $2.981 \mathrm{mmHg}$ $\left(n=14,1.323-4.639, p<0.001 ; \mathrm{I}^{2}=45.343, p=0.034\right)[25,35,44,45,47,48,50,51,53,54,56]$. In community health centers, the WMD was $3.512 \mathrm{mmHg}(n=12,1.651-5.373, p<0.001$; $\left.\mathrm{I}^{2}=31.670, p<0.001\right)[27-30,42,57,61]$, and the WMD at hospital level was $6.333 \mathrm{mmHg}$ $\left(n=22,4.750-7.917, p<0.001 ; \mathrm{I}^{2}=73.401, p<0.001\right)[26,31-34,41,43,46,49,52,55,58-60]$.

\subsubsection{Frequency of Remote Transmission of Blood Pressure Data}

In the primary studies in which the frequency of remote BP transmission was reported, when BP information was transmitted daily, the WMD was $5.881 \mathrm{mmHg}(n=13$, $\left.3.898-7.864, p<0.001 ; \mathrm{I}^{2}=14.635, p<0.001\right)[27,34,49,53-55,59-61]$. For weekly BP transmission, the WMD was $4.024\left(n=15,2.641-5.406, p<0.001 ; \mathrm{I}^{2}=54.610, p<0.001\right)[28,30$, $32,42,43,45,47,52,56-58]$. For biweekly BP transmission, the WMD was $3.941 \mathrm{mmHg}(n=4$, $1.428-6.454, p<0.001 ; \mathrm{I}^{2}=0.000$ ). For monthly BP transmission, the WMD was $1.803 \mathrm{mmHg}$ $\left(n=6,-0.234-3.841, p=0.083 ; \mathrm{I}^{2}=21.639, p=0.056\right)[26,35,41,50]$.

\section{Discussion}

The development of healthcare infrastructure and physicians' preference for practice in an urban setting implies higher accessibility to healthcare and higher patient satisfaction regarding healthcare [65]. However, the COVID-19 pandemic has raised concerns regarding face-to-face care in cities being a potential infection route between healthcare professionals and patients. In this study, data published since September 2018 were included and integrated with data from previous studies to undertake an updated analysis.

Compared with UC, RBPM for urban-dwelling patients with hypertension was found to significantly reduce SBP and DBP in both statistical and clinical terms, while improving the rate of BP control. Following RBPM, SBP and DBP WMDs decreased by $4.464 \mathrm{mmHg}$ and $2.075 \mathrm{mmHg}$, respectively, compared with UC. This change, observed through quantitative data, showed a greater margin of decrease than reported in a previous meta-analysis 
(SBP, $3.482 \mathrm{mmHg}$; DBP, $1.638 \mathrm{mmHg}$ ) [20]. Moreover, according to the temporal interval, the decrease in SBP (1.515 vs. $4.719 \mathrm{mmHg}$ ) and DBP (2.059 vs. $2.438 \mathrm{mmHg}$ ) in phase III was significantly greater than that in phase I. Therefore, we consider that the demand for RBPM has increased in line with technological advancements, the increased use of mobile devices, and the acceptance of new technologies [66].

RBPM is frequently used in pilot projects preceding the full launch of telemedicine, as it is relatively simple and cost-effective compared with other types of telemedicine. However, reports on the effect of RBPM on the rate of BP control have been inconsistent across numerous previous studies [14]. In this study, where additional data were comprehensively analyzed to extend the meta-analysis, RBPM led to an approximately $20 \%$ higher rate of BP control than UC. This is a greater magnitude of improvement than the $13 \%$ figure reported in a previous analysis [20]. Considering that the rate of BP control is $<50 \%$ in traditional face-to-face care, even in countries with advanced healthcare systems, an improvement of $20 \%$ is indicative of a highly significant contribution to the prevention of CVD [67].

The ultimate objective behind attempts to lower and control BP in patients with hypertension and to bring it closer to a target BP is to reduce the incidence of CVD. However, in the meta-analysis in this study, data were not analyzed in relation to cardiovascular (CV) events because the included RCTs primarily showed outcomes that targeted changes in BP or the rate of BP control, not CV events. Nevertheless, the effect of RBPM on CV events in urban-dwelling patients with hypertension can be conjectured based on the results of previous studies. In a previous large-scale meta-analysis on prospective monitoring, including randomized, controlled, placebo trials or anti-hypertensive studies, a decrease of 2-3 mmHg in SBP in patients with a moderate risk of CVD was shown to cause a $10 \%$ reduction in CV mortality and a 20-30\% reduction in major adverse CV events [2,68-70]. Thus, the observed decrease in SBP of $4.464 \mathrm{mmHg}$ in this study, when the WMD was compared between UC and RBPM, is clinically significant and potentially contributes to reducing $\mathrm{CV}$ events.

The effect size of the primary outcomes was set as the SMD and, as it showed moderateto-high heterogeneity $\left(\mathrm{I}^{2}=70.908 \% ; p<0.001\right)$, a subgroup analysis was performed (Appendix E, Table A1). First, the analysis according to city size (based on population size) showed that the effect of RBPM was greater in cities with a population of $\geq 1$ million (SBP, $\left.3.860 \mathrm{mmHg}, p<0.001 ; \mathrm{I}^{2}=0.000, p=0.478\right)$ than in small-to-medium cities with a smaller population, although within-study heterogeneity was high $\left(\mathrm{I}^{2}=82.177, p<0.001\right)$. The effect of RBPM in reducing SBP was statistically significant compared with UC, irrespective of city size. The rate of BP control also showed greater effects in large cities (RR, 1.268; $p<0.001)$ than in small-to-medium-sized cities ( $\mathrm{RR}, 1.157 ; p=0.094)$. In a previous literature review, the intervention effect was found to be smaller in larger cities (large city, $3.229 \mathrm{mmHg}$ vs. small-to-medium city, $3.765 \mathrm{mmHg}$ ), where the difference was considered to be associated with the difference in technological utility based on acceptance [66]. In particular, there was a sudden rise in demand for telemedicine to avoid the transmission of infectious diseases in response to the COVID-19 pandemic in 2020 [71,72].

Second, subgroup analysis was also performed according to urban MUAs in terms of healthcare accessibility. The decrease in BP in relation to RBPM in non-MUAs was $5.224 \mathrm{mmHg}\left(\mathrm{I}^{2}=73.152 \%, p<0.001\right)$, indicating a greater effect of RBPM in reducing SBP compared with MUAs $\left(3.213 \mathrm{mmHg}, p<0.001 ; \mathrm{I}^{2}=48.904 \%, p=0.012\right)$. The extent to which the level of within-study heterogeneity affects the summary effect size remains unclear, but the results of the analysis provided supporting evidence for determining the overall effect. Although a precise reason for this result could not be identified in this study, the following factors may be considered: changes in attitudes towards the use of mobile devices and chronic disease management and changes in economic lifestyle related to reduced opportunities for healthcare. These results may be used as evidence by healthcare policy-makers to support the need for differentiated policies for the supply of telemedicine in urban settings. 
Third, a subgroup analysis was also performed concerning the duration of intervention. No optimal schedule has been established for the period of management of hypertension based on RBPM and the frequency of remote transmission of data $[47,73]$. Despite slight differences in the magnitude of reduction in SBP, RBPM in this study showed a consistent effect of reducing SBP, regardless of duration. Nonetheless, as the intervention duration increased, the level of BP reduction decreased. The reason for such a decrease could not be clearly identified, but possible causes may be fatigue, indifference, and inadequate level of perceived utility due to the prolonged performance of the intervention [73,74]. However, considering that it is essential to achieve a target BP as early as possible in patients with hypertension to prevent CVD, the effect of RBPM on the early outcome of BP reduction may be emphasized for its use in practice. The optimal duration of RBPM should be limited to a short period of time due to hypertension being a chronic disease requiring long-term management.

Fourth, in this updated study, subgroup analysis was undertaken according to the setting where RBPM was mainly implemented. Accordingly, when the intervention was performed at a tertiary hospital, RBPM had a significant reduction in $\mathrm{BP}(6.33 \mathrm{mmHg}$, $\left.p<0.001 ; \mathrm{I}^{2}=73.401 \%, p<0.001\right)$. The same numerical comparison was not compared in each group and, in the case of hospitals, its size was not analyzed separately; however, the results were statistically significant and included a sufficient number of studies to support the results; therefore, the significance of the results should not be ignored. The reason that RBPM had a higher BP lowering effect in tertiary medical institutions than in primary medical institutions may be due to the greater financial and human resource capacity in tertiary medical institutions [75].

Finally, this study observed the effect of RBPM with respect to the frequency of transmission of BP data. In the case of daily transmission, the WMD decreased by $5.881 \mathrm{mmHg}$. In contrast, in the case of monthly transmission, a decrease of $1.803 \mathrm{mmHg}$ was observed. Some conflicting studies show that the higher the frequency of remote transmission, the lower the BP reduction effect $[60,61]$. However, in our study on cities, the longer the transmission interval, the lower the effect.

In previous meta-analyses, the number of studies conducted in urban settings was insufficient, and no study showed a change according to temporal progression. In this updated research, we included a comparison of the average effect over time, which was not covered in previous studies, and the effect according to the frequency of setting and data teletransmission. In particular, in our previous meta-analysis, it was reported that the effect of RBPM on patients with hypertension in metropolitan cities was not as large as that in small and medium cities. However, in this updated study, we found that the decrease in SBP and DBP was large in cities with a population of $\geq 1$ million. Therefore, this study addressed the limitations of previous studies. Advancements in telecommunication technology have led to increased use of remote monitoring in healthcare [76]. In situations where physical distancing is emphasized, such as in the case of COVID-19, it is essential to assess the effects of RBPM in an urban setting [77]. To our knowledge, this study is the first meta-analysis to assess the effects of RBPM in urban-dwelling patients with hypertension from RBPM inception to the end of November 2020, including during the COVID-19 pandemic period, and these comprehensive results may provide a clinical basis for developing future healthcare policies.

In this study, a structured formula was applied, and a transparent process was followed to analyze RCTs with a high level of evidence. However, this study had some limitations. First, although the final studies were selected through a structured search using reliable databases, there may have been a language barrier. No outlier was found to have an influence on the summary effect size through the "one-study removed" sensitivity test method and a cumulative meta-analysis; however, selecting articles in different languages may have prevented adequate accounting for errors. Although most abstracts included in the search were written in English, the collected data may not have been sufficient. To overcome this limitation, multiple languages need to be set in the search with a wider scope 
to include gray literature. Second, the number of small-sized articles was insufficient to test for publication errors. Egger's test for the results in this study was used to determine combined two-tailed $p$-value significance, and the number of articles with a nil result in terms of a $95 \%$ CI was as high as 2898 , which increased reliability. Nevertheless, there remained the possibility of publication errors. This limitation could be addressed through including a larger number of small-sized articles. Third, as the studies included in this meta-analysis varied in terms of the period when they were conducted, the criteria for target BP reflected in the rate of BP control may also have varied. Thus, further studies should set a clear BP target for collecting and synthesizing the data to produce more accurate results. Fourth, the authors categorized time intervals to compare the average SBP according to time interval and to quantify the results, which involved dividing the studies according to time based on the year 2000, when internet use expanded globally, and making simple comparisons at 10-year intervals thereafter. However, distinctions between time intervals may have been unclear. Although it is not possible to clearly divide the development time of telemedicine technology, we consider that the timeframe could be set more precisely based on historical developments in mobile communication technology and telemedicine. Finally, we examined trends in the effect of RBPM over time through categorizing studies based on their publication dates to indicate the temporal association with COVID-19. However, since differences between the actual dates of research and publication dates are possible, a future study should clarify the dates during which studies were conducted or include more studies published after the onset of the COVID-19 pandemic to address this limitation.

\section{Conclusions}

Our study findings indicated that RBPM for urban-dwelling patients with hypertension was a practical and clinically effective means of reducing office BP. As the cumulative analysis shows, a consistent and clear effect was found in terms of reduction in office SBP following RBPM according to the temporal progress of the primary studies included in this study; an identical trend was found for 2020.

Based on the primary findings, the effects were classified according to intervention duration, city size, setting, frequency of remote monitoring of BP data, and urban MUAs, and it is anticipated that the implementation of specific policies in relation to these factors would more effectively guide the application of efficient and successful urban remote monitoring. Future studies should analyze more specific variables and include a greater number of studies to obtain more reliable results.

Supplementary Materials: The following are available online at https:/ /www.mdpi.com/article/10 .3390 /ijerph182010583/s1, Table S1: PRISMA 2020 Checklist.

Author Contributions: Conceptualization, W.-S.C. and J.-H.S.; methodology, W.-S.C.; software, W.S.C.; validation, W.-S.C., S.-H.P., J.P. and J.-H.S.; formal analysis, W.-S.C.; investigation, S.-H.P. and J.-H.S.; resources, W.-S.C.; data curation, J.P.; writing—original draft preparation, S.-H.P. and J.-H.S.; writing-review and editing, W.-S.C. and S.-H.P.; visualization, J.P. and W.-S.C.; supervision, W.-S.C. All authors have read and agreed to the published version of the manuscript.

Funding: This research received no external funding.

Institutional Review Board Statement: Not applicable.

Informed Consent Statement: Not applicable.

Data Availability Statement: Not applicable.

Conflicts of Interest: The authors declare no conflict of interest. 


\section{Appendix A. Searching Strategy via Cochrane Library}

1. MeSH descriptor: [Hypertension] explode all trees

2. hypertensi* OR high blood pressure

3. $\mathrm{OR} / 1,2$

4. MeSH descriptor: [Urban population] explode all trees

5. MeSH descriptor: [Urban health] explode all trees urban health [Mesh]

6. MeSH descriptor: [Urban health services] explode all trees

7. MeSH descriptor: [Cities] explode all trees

8. urban* OR city OR cities OR central cit*

9. $\mathrm{OR} / 4-8$

10. AND $/ 3,10$

11. MeSH descriptor: [Telemedicine] explode all trees

12. MeSH descriptor: [Telemetry] explode all trees

13. MeSH descriptor: [Blood pressure monitoring, ambulatory] explode all trees

14. telemedicine OR telemetry OR telenurs* OR telemonitor* OR eHealth OR telehealth OR remote monitor* OR technolog* OR telephone OR smartphone OR internet

15. OR/12-15

16. AND/11,16

17. randomised controlled trial OR randomized controlled

18. controlled clinical trial

19. randomised [tiab] OR randomized [tiab]

20. 2placebo [tiab]

21. drug therapy [sh]

22. groups [tiab]

23. clinical trials as topic [tiab]

24. randomly [tiab]

25. trial [tiab]

26. OR/18-26

27. 27 NOT cluster randomized controlled trials

28. 28 NOT cross over study

29. AND/17,29 


\section{Appendix B. Sensitivity Test Based on a "One-Study Removed" Approach}

\begin{tabular}{|c|c|c|c|c|c|c|}
\hline \multirow[t]{2}{*}{ Author (year) (ref.) } & \multirow[t]{2}{*}{ Outcome } & \multicolumn{5}{|c|}{ Statistics with study removed } \\
\hline & & $\begin{array}{c}\text { Lower } \\
\text { limit }\end{array}$ & $\begin{array}{c}\text { Upper } \\
\text { limit }\end{array}$ & Z-Value & Point & $\mathrm{p}$-Value \\
\hline Artinian,2007A & SBP & 0.367 & 0.779 & 5.456 & 0.573 & 0.000 \\
\hline Artinian,2007B & SBP & 0.375 & 0.786 & 5.536 & 0.580 & 0.000 \\
\hline Bosworth,2007A & SBP & 0.340 & 0.715 & 5.519 & 0.528 & 0.000 \\
\hline Bosworth,2007B & SBP & 0.314 & 0.630 & 5.864 & 0.472 & 0.000 \\
\hline Bosworth,2007C & SBP & 0.339 & 0.709 & 5.541 & 0.524 & 0.000 \\
\hline Bosworth,2011 & SBP & 0.374 & 0.784 & 5.537 & 0.579 & 0.000 \\
\hline Bove,2013 & SBP & 0.373 & 0.782 & 5.539 & 0.578 & 0.000 \\
\hline Carrasco,2008 & SBP & 0.373 & 0.783 & 5.522 & 0.578 & 0.000 \\
\hline Cicolini,2013A & SBP & 0.376 & 0.784 & 5.570 & 0.580 & 0.000 \\
\hline Cicolini,2013B & SBP & 0.361 & 0.769 & 5.436 & 0.565 & 0.000 \\
\hline Friedman,1996 & SBP & 0.379 & 0.787 & 5.595 & 0.583 & 0.000 \\
\hline Green,2008 & SBP & 0.370 & 0.786 & 5.457 & 0.578 & 0.000 \\
\hline Hill,1999 & SBP & 0.376 & 0.783 & 5.575 & 0.579 & 0.000 \\
\hline Hoffmann,2017 & SBP & 0.375 & 0.786 & 5.536 & 0.581 & 0.000 \\
\hline Ionov, 2020 & SBP & 0.348 & 0.746 & 5.391 & 0.547 & 0.000 \\
\hline Kerry,2013A & SBP & 0.376 & 0.786 & 5.547 & 0.581 & 0.000 \\
\hline Kerry,2013B & SBP & 0.375 & 0.786 & 5.536 & 0.580 & 0.000 \\
\hline Kim KB,2014A & SBP & 0.366 & 0.779 & 5.432 & 0.572 & 0.000 \\
\hline Kim KB,2014B & SBP & 0.372 & 0.784 & 5.492 & 0.578 & 0.000 \\
\hline Logan,2012 & SBP & 0.367 & 0.773 & 5.497 & 0.570 & 0.000 \\
\hline Madsen,2008 & SBP & 0.374 & 0.783 & 5.542 & 0.578 & 0.000 \\
\hline McMahon,2005 & SBP & 0.376 & 0.781 & 5.593 & 0.578 & 0.000 \\
\hline Mohsen,2020A & SBP & 0.358 & 0.762 & 5.424 & 0.560 & 0.000 \\
\hline Mohsen,2020B & SBP & 0.338 & 0.734 & 5.308 & 0.536 & 0.000 \\
\hline Neumann,2011 & SBP & 0.365 & 0.769 & 5.490 & 0.567 & 0.000 \\
\hline Pan,2018A & SBP & 0.369 & 0.775 & 5.514 & 0.572 & 0.000 \\
\hline Pan,2018B & SBP & 0.369 & 0.776 & 5.518 & 0.572 & 0.000 \\
\hline Parati,2009 & SBP & 0.379 & 0.788 & 5.597 & 0.583 & 0.000 \\
\hline Park MJ, 2012 & SBP & 0.365 & 0.771 & 5.495 & 0.568 & 0.000 \\
\hline Park MJ,2009 & SBP & 0.351 & 0.753 & 5.378 & 0.552 & 0.000 \\
\hline Rahmani Pour,2020A & SBP & 0.369 & 0.774 & 5.538 & 0.572 & 0.000 \\
\hline Rahmani Pour,2020B & SBP & 0.361 & 0.765 & 5.466 & 0.563 & 0.000 \\
\hline Rahmani Pour,2020C & SBP & 0.378 & 0.783 & 5.630 & 0.581 & 0.000 \\
\hline Rahmani Pour,2020D & SBP & 0.372 & 0.777 & 5.567 & 0.575 & 0.000 \\
\hline Rubinstein, 2016A & SBP & 0.375 & 0.789 & 5.514 & 0.582 & 0.000 \\
\hline Rubinstein, 2016B & SBP & 0.377 & 0.790 & 5.546 & 0.584 & 0.000 \\
\hline Shea,2006 & SBP & 0.372 & 0.789 & 5.448 & 0.580 & 0.000 \\
\hline Wakefield,2011 & SBP & 0.372 & 0.781 & 5.532 & 0.576 & 0.000 \\
\hline Wakefield,2014 & SBP & 0.376 & 0.782 & 5.593 & 0.579 & 0.000 \\
\hline Zha,2020A & SBP & 0.370 & 0.774 & 5.557 & 0.572 & 0.000 \\
\hline Zha,2020B & SBP & 0.364 & 0.768 & 5.498 & 0.566 & 0.000 \\
\hline & & 0.369 & 0.768 & 5.585 & 0.568 & 0.000 \\
\hline
\end{tabular}
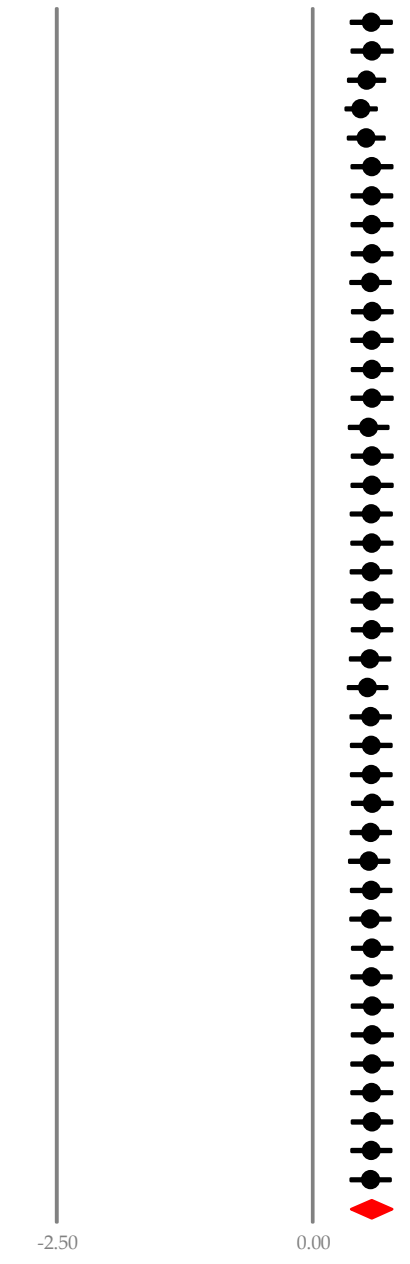

Favors UC

Favors RBPM

Figure A1. Note: point estimate of individual study $(\bullet)$, summary effect size $(\bullet)$; SBP, systolic blood pressure; UC, usual care; RBPM, remote blood pressure monitoring. 


\section{Appendix C. Cumulative Meta-Analysis of RBPM According to the SMD of SBP}

\begin{tabular}{|c|c|c|c|c|c|c|c|}
\hline \multirow[t]{2}{*}{ Author (year) (ref.) } & \multirow[t]{2}{*}{ Outcome } & \multirow[t]{2}{*}{ Time point } & \multicolumn{5}{|c|}{ Cumulative statistics } \\
\hline & & & Point & $\begin{array}{c}\text { Lower } \\
\text { limit }\end{array}$ & $\begin{array}{c}\text { Upper } \\
\text { limit }\end{array}$ & Z-Value & $p$-Value \\
\hline Friedman,1996 & SBP & 1996.000 & 0.023 & -0.217 & 0.263 & 0.189 & 0.850 \\
\hline Hill,1999 & SBP & 1999.000 & 0.070 & -0.121 & 0.261 & 0.714 & 0.475 \\
\hline McMahon,2005 & SBP & 2005.000 & 0.082 & -0.094 & 0.259 & 0.915 & 0.360 \\
\hline Shea,2006 & SBP & 2006.000 & 0.121 & 0.007 & 0.236 & 2.075 & 0.038 \\
\hline Artinian,2007A & SBP & 2007.000 & 0.184 & 0.050 & 0.318 & 2.687 & 0.007 \\
\hline Artinian,2007B & SBP & 2007.000 & 0.175 & 0.069 & 0.280 & 3.247 & 0.001 \\
\hline Bosworth,2007A & SBP & 2007.000 & 0.442 & -0.031 & 0.916 & 1.830 & 0.067 \\
\hline Bosworth,2007B & SBP & 2007.000 & 0.912 & 0.166 & 1.658 & 2.396 & 0.017 \\
\hline Bosworth,2007C & SBP & 2007.000 & 1.056 & 0.315 & 1.797 & 2.793 & 0.005 \\
\hline Carrasco,2008 & SBP & 2008.000 & 0.971 & 0.309 & 1.634 & 2.873 & 0.004 \\
\hline Green,2008 & SBP & 2008.000 & 0.902 & 0.322 & 1.482 & 3.048 & 0.002 \\
\hline Madsen,2008 & SBP & 2008.000 & 0.843 & 0.308 & 1.378 & 3.086 & 0.002 \\
\hline Parati,2009 & SBP & 2009.000 & 0.778 & 0.281 & 1.275 & 3.067 & 0.002 \\
\hline Park MJ,2009 & SBP & 2009.000 & 0.815 & 0.335 & 1.294 & 3.329 & 0.001 \\
\hline Bosworth,2011 & SBP & 2011.000 & 0.771 & 0.324 & 1.218 & 3.380 & 0.001 \\
\hline Neumann,2011 & SBP & 2011.000 & 0.763 & 0.333 & 1.194 & 3.478 & 0.001 \\
\hline Wakefield,2011 & SBP & 2011.000 & 0.734 & 0.327 & 1.141 & 3.531 & 0.000 \\
\hline Logan,2012 & SBP & 2012.000 & 0.722 & 0.331 & 1.112 & 3.624 & 0.000 \\
\hline Park MJ, 2012 & SBP & 2012.000 & 0.715 & 0.338 & 1.093 & 3.719 & 0.000 \\
\hline Bove,2013 & SBP & 2013.000 & 0.690 & 0.331 & 1.049 & 3.769 & 0.000 \\
\hline Cicolini,2013A & SBP & 2013.000 & 0.663 & 0.320 & 1.006 & 3.786 & 0.000 \\
\hline Cicolini,2013B & SBP & 2013.000 & 0.664 & 0.336 & 0.993 & 3.961 & 0.000 \\
\hline Kerry,2013A & SBP & 2013.000 & 0.639 & 0.326 & 0.951 & 4.007 & 0.000 \\
\hline Kerry,2013B & SBP & 2013.000 & 0.616 & 0.319 & 0.914 & 4.063 & 0.000 \\
\hline Kim KB,2014A & SBP & 2014.000 & 0.608 & 0.327 & 0.890 & 4.234 & 0.000 \\
\hline Kim KB,2014B & SBP & 2014.000 & 0.593 & 0.324 & 0.861 & 4.332 & 0.000 \\
\hline Wakefield,2014 & SBP & 2014.000 & 0.576 & 0.315 & 0.838 & 4.321 & 0.000 \\
\hline Rubinstein, 2016A & SBP & 2016.000 & 0.557 & 0.308 & 0.805 & 4.392 & 0.000 \\
\hline Rubinstein, 2016B & SBP & 2016.000 & 0.537 & 0.299 & 0.774 & 4.430 & 0.000 \\
\hline Hoffmann,2017 & SBP & 2017.000 & 0.522 & 0.293 & 0.751 & 4.475 & 0.000 \\
\hline Pan,2018A & SBP & 2018.000 & 0.519 & 0.296 & 0.743 & 4.556 & 0.000 \\
\hline Pan,2018B & SBP & 2018.000 & 0.516 & 0.298 & 0.734 & 4.631 & 0.000 \\
\hline Ionov,2020 & SBP & 2020.000 & 0.543 & 0.324 & 0.762 & 4.859 & 0.000 \\
\hline Mohsen,2020A & SBP & 2020.000 & 0.554 & 0.338 & 0.769 & 5.035 & 0.000 \\
\hline Mohsen,2020B & SBP & 2020.000 & 0.591 & 0.374 & 0.808 & 5.337 & 0.000 \\
\hline Rahmani Pour,2020A & SBP & 2020.000 & 0.587 & 0.373 & 0.801 & 5.384 & 0.000 \\
\hline Rahmani Pour,2020B & SBP & 2020.000 & 0.592 & 0.382 & 0.803 & 5.507 & 0.000 \\
\hline Rahmani Pour,2020C & SBP & 2020.000 & 0.579 & 0.371 & 0.787 & 5.460 & 0.000 \\
\hline Rahmani Pour,2020D & SBP & 2020.000 & 0.573 & 0.368 & 0.778 & 5.479 & 0.000 \\
\hline Zha,2020A & SBP & 2020.000 & 0.569 & 0.367 & 0.772 & 5.509 & 0.000 \\
\hline Zha,2020B & SBP & 2020.000 & 0.572 & 0.372 & 0.773 & 5.598 & 0.000 \\
\hline & & & 0.572 & 0.372 & 0.773 & 5.598 & 0.000 \\
\hline
\end{tabular}
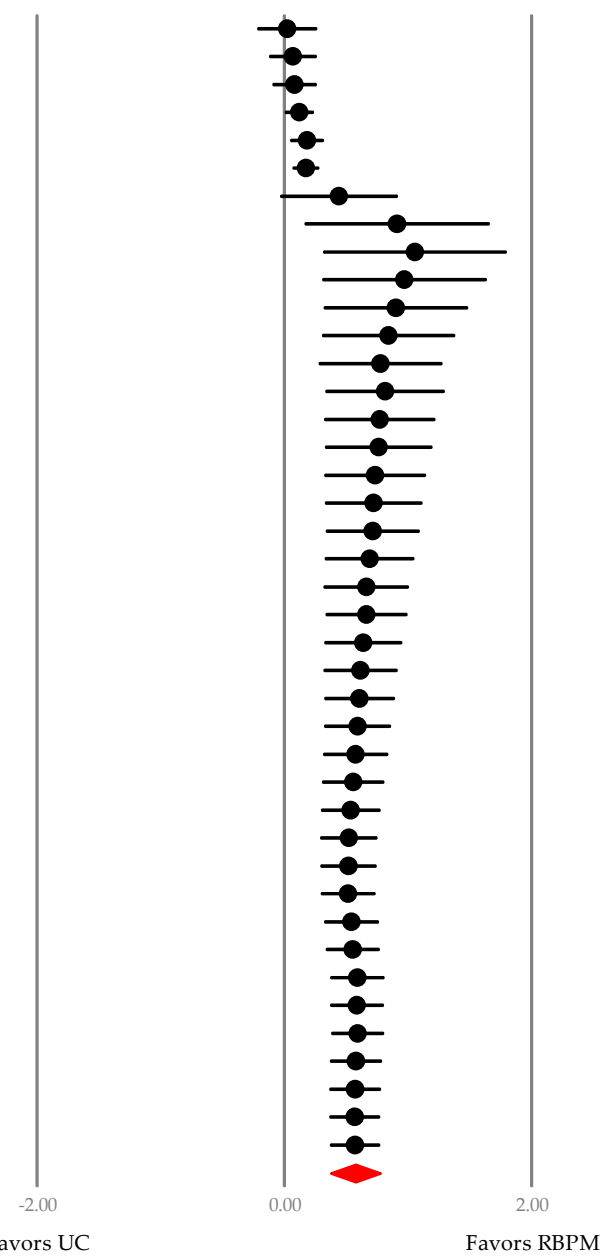

Favors UC

Favors RBPM

Figure A2. Note: point estimate of individual study excluding each individual study $(\bullet)$, summary effect size $(\bullet)$; SBP, systolic blood pressure; SMD, standardized mean difference; UC, usual care; RBPM, remote blood pressure monitoring. 
Appendix D. Meta-Regression of Risk Ratio According to RBPM Duration

\section{Regression of Duration of intervention, months on Log risk ratio}

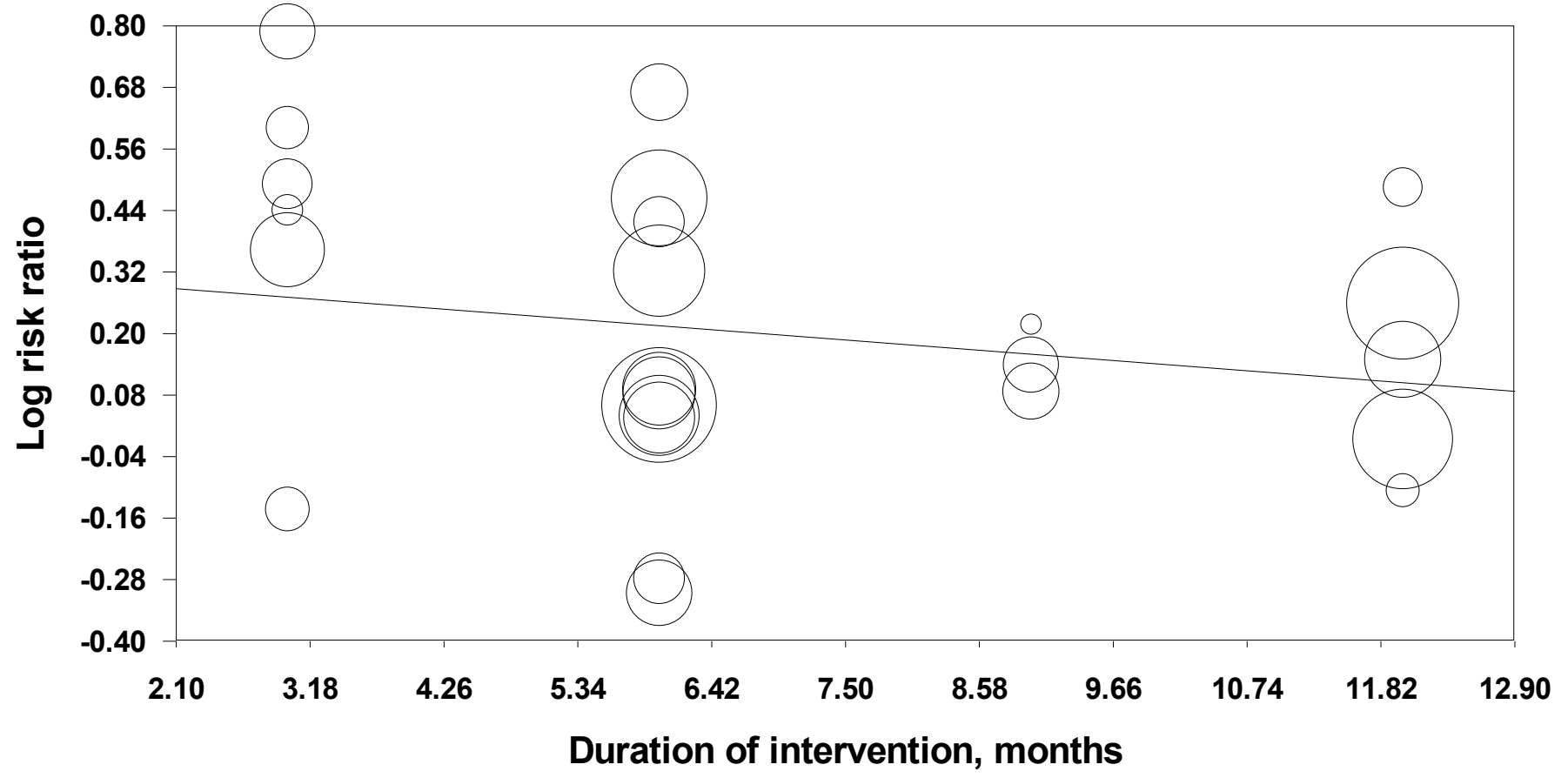

Figure A3. Note: point estimate of individual study $(\bigcirc)$; RBPM, remote blood pressure monitoring.

Appendix E. Subgroup Analysis

Table A1. CI, confidence interval; FEM, fixed effects model; SBP, systolic blood pressure; WMD, weighted mean difference.

\begin{tabular}{|c|c|c|c|c|}
\hline Category & Number of Studies & $\begin{array}{l}\text { Summary WMD of } \\
\text { SBP, mmHg }(95 \% \text { CI })\end{array}$ & $\begin{array}{c}\text { Heterogeneity, } \mathrm{I}^{2}(\%) \\
\text { Using an FEM } \\
(p \text {-Value })\end{array}$ & $\begin{array}{c}\text { Heterogeneity, } \\
\text { Tau-Squared }\left(\tau^{2}\right) \\
\text { Using an FEM }\end{array}$ \\
\hline Overall & 48 & $4.464(3.371-5.556)$ & $70.908(p<0.001)$ & 9.200 \\
\hline \multicolumn{5}{|l|}{ City size (population) } \\
\hline$<1$ million & 22 & $3.860(2.271-5.450)$ & $0.000(p=0.478)$ & 0.000 \\
\hline$>1$ million & 26 & $5.056(3.503-6.609)$ & $82.177(p<0.001)$ & 17.368 \\
\hline \multicolumn{5}{|l|}{$\begin{array}{l}\text { Medically underserved } \\
\text { areas }\end{array}$} \\
\hline Underserved & 17 & $3.213(1.521-4.905)$ & $48.904(p=0.012)$ & 2.793 \\
\hline Not underserved & 31 & $5.224(3.878-6.569)$ & $73.152(p<0.001)$ & 12.943 \\
\hline \multicolumn{5}{|l|}{ Duration (month) } \\
\hline$\leq 3$ & 15 & 6.198 (4.019-8.377) & $70.060(p<0.001)$ & 14.069 \\
\hline 6 & 14 & $4.479(2.524-6.433)$ & $84.562(p<0.001)$ & 17.240 \\
\hline 9 & 4 & $2.116(-1.816-6.048)$ & $0.000(p=0.752)$ & 0.000 \\
\hline 12 & 12 & $3.436(1.281-5.591)$ & $34.656(p=0.113)$ & 1.646 \\
\hline \multicolumn{5}{|l|}{ Setting } \\
\hline Primary care clinic & 14 & $2.981(1.323-4.639)$ & $45.243(p=0.034)$ & 1.989 \\
\hline $\begin{array}{l}\text { Community health } \\
\text { center }\end{array}$ & 12 & $3.512(1.651-5.373)$ & $31.670(p=0.138)$ & 1.883 \\
\hline
\end{tabular}


Table A1. Cont.

\begin{tabular}{ccccc}
\hline Category & Number of Studies & $\begin{array}{c}\text { Summary WMD of } \\
\text { SBP, } \mathbf{m m H g} \mathbf{( 9 5 \%} \text { CI) }\end{array}$ & $\begin{array}{c}\text { Heterogeneity, } \mathbf{I}^{\mathbf{2}}(\mathbf{\%}) \\
\text { Using an FEM } \\
(\boldsymbol{p} \text {-Value) }\end{array}$ & $\begin{array}{c}\text { Heterogeneity, } \\
\text { Tau-Squared }\left(\boldsymbol{\tau}^{\mathbf{2}}\right) \\
\text { Using an FEM }\end{array}$ \\
\hline $\begin{array}{c}\text { Hospital } \\
\begin{array}{c}\text { Frequency of data } \\
\text { transmission }\end{array}\end{array}$ & 22 & $6.333(4.750-7.917)$ & $73.401(p<0.001)$ & 17.133 \\
\hline Daily & 13 & $5.881(3.898-7.864)$ & $14.635(p=0.297)$ & 1.637 \\
\hline Weekly & 15 & $4.024(2.641-5.406)$ & $53.610(p=0.007)$ & 4.505 \\
\hline Bi-weekly & 4 & $3.941(1.428-6.454)$ & $0.000(p=0.622)$ & 0.000 \\
\hline Monthly & 6 & $1.803(-0.234-3.841)$ & $21.639(p=0.271)$ & 0.552 \\
\hline
\end{tabular}

\section{References}

1. Kishore, S.P.; Heller, D.J.; Vasan, A. Beyond hypertension: Integrated cardiovascular care as a path to comprehensive primary care. B. World Health Organ. 2018, 96, 219-221. [CrossRef] [PubMed]

2. Lewington, S.; Clarke, R.; Qizilbash, N.; Peto, R.; Collins, R. Age-specific relevance of usual blood pressure to vascular mortality: A meta-analysis of individual data for one million adults in 61 prospective studies. Lancet 2002, 360, 1903-1913. [PubMed]

3. Chow, C.K.; Teo, K.K.; Rangarajan, S.; Islam, S.; Gupta, R.; Avezum, A.; Bahonar, A.; Chifamba, J.; Dagenais, G.; Diaz, R.; et al. Prevalence, awareness, treatment, and control of hypertension in rural and urban communities in high-, middle-, and low-income countries. JAMA 2013, 310, 959-968. [CrossRef]

4. Egan, B.M.; Li, J.; Hutchison, F.N.; Ferdinand, K.C. Hypertension in the United States, 1999 to 2012: Progress toward Healthy People 2020 goals. Circulation 2014, 130, 1692-1699. [CrossRef]

5. Williams, B.; Mancia, G.; Spiering, W.; Rosei, E.A.; Azizi, M.; Burnier, M.; Clement, D.L.; Coca, A.; de Simone, G.; Dominiczak, A.; et al. 2018 ESC/ESH Guidelines for the management of arterial hypertension. Eur. Heart J. 2018, 39, 3021-3104. [CrossRef]

6. Whelton, P.K.; Carey, R.M.; Aronow, W.S.; Casey, D.E., Jr.; Collins, K.J.; Himmelfarb, C.D.; DePalma, S.M.; Gidding, S.; Jamerson, K.A.; Jones, D.W.; et al. 2017 ACC/AHA/AAPA/ABC/ACPM/AGS/APhA/ASH/ASPC/NMA/PCNA Guideline for the Prevention, Detection, Evaluation, and Management of High Blood Pressure in Adults: A Report of the American College of Cardiology/American Heart Association Task Force on Clinical Practice Guidelines. Circulation 2018, 138, e484-e594. [PubMed]

7. Ward, A.M.; Takahashi, O.; Stevens, R.; Heneghan, C. Home measurement of blood pressure and cardiovascular disease: Systematic review and meta-analysis of prospective studies. J. Hypertens. 2012, 30, 449-456. [CrossRef]

8. McManus, R.J.; Mant, J.; Bray, E.P.; Holder, R.; Jones, M.I.; Greenfield, S.; Kaambwa, B.; Banting, M.; Bryan, S.; Little, P.; et al. Telemonitoring and self-management in the control of hypertension (TASMINH2): A randomised controlled trial. Lancet 2010, 376, 163-172. [CrossRef]

9. McManus, R.; Mant, J.; Haque, M.; Bray, E.P.; Bryan, S.; Greenfield, S.; Jones, M.I.; Jowett, S.; Little, P.; Peñaloza, C.; et al. Effect of self-monitoring and medication self-titration on systolic blood pressure in hypertensive patients at high risk of cardiovascular disease: The TASMIN-SR randomized clinical trial. JAMA 2014, 312, 799-808. [CrossRef]

10. Tucker, K.L.; Sheppard, J.P.; Stevens, R.; Bosworth, H.B.; Bove, A.; Bray, E.P.; Earle, K.; George, J.; Godwin, M.; Green, B.B.; et al. Self-monitoring of blood pressure in hypertension: A systematic review and individual patient data meta-analysis. PLoS Med. 2017, 14, e1002389. [CrossRef]

11. Kim, Y.N.; Shin, D.G.; Park, S.; Lee, C.H. Randomized clinical trial to assess the effectiveness of remote patient monitoring and physician care in reducing office blood pressure. Hypertens. Res. 2015, 38, 491-497. [CrossRef]

12. Green, B.B.; Anderson, M.L.; Cook, A.J.; Catz, S.; Fishman, P.A.; McClure, J.B.; Reid, R.J. e-Care for heart wellness: A feasibility trial to decrease blood pressure and cardiovascular risk. Am. J. Prev. Med. 2014, 46, 368-377. [CrossRef] [PubMed]

13. Parati., G.; Ochoa, J.E.; Postel-Vinay, N.; Pellegrini, D.; Torlasco, C.; Omboni, S.; McManus, R. Home Blood Pressure Telemonitoring: Conventional Approach and Perspectives from Mobile Health Technology. In Home Blood Pressure Monitoring; Springer: Cham, Switzerland, 2019; pp. 103-119. [CrossRef]

14. Omboni, S.; McManus, R.J.; Bosworth, H.B.; Chappell, L.C.; Green, B.B.; Kario, K.; Logan, A.G.; Magid, D.J.; McKinstry, B.; Margolis, K.L.; et al. Evidence and Recommendations on the Use of Telemedicine for the Management of Arterial Hypertension: An International Expert Position Paper. Hypertension 2020, 76, 1368-1383. [CrossRef] [PubMed]

15. Lu, X.; Yang, H.; Xia, X.; Lu, X.; Lin, J.; Liu, F.; Gu, D. Interactive Mobile Health Intervention and Blood Pressure Management in Adults. Hypertension 2019, 74, 697-704. [CrossRef] [PubMed]

16. Duan, Y.; Xie, Z.; Dong, F.; Wu, Z.; Lin, Z.; Sun, N.; Xu, J. Effectiveness of home blood pressure telemonitoring: A systematic review and meta-analysis of randomised controlled studies. J. Hum. Hypertens. 2017, 31, 427-437. [CrossRef]

17. United Nations Department of Economic and Social Affairs. World Urbanization Prospects: The 2018 Revision; online edition; United Nations Department of Economic and Social Affairs: New York, NY, USA, 2018. 
18. Omboni, S.; Gazzola, T.; Carabelli, G.; Parati, G. Clinical usefulness and cost effectiveness of home blood pressure telemonitoring: Meta-analysis of randomized controlled studies. J. Hypertens. 2013, 31, 455-467, discussion 67-68. [CrossRef]

19. Choi, W.S.; Park, J.; Choi, J.Y.B.; Yang, J.S. Stakeholders' resistance to telemedicine with focus on physicians: Utilizing the Delphi technique. J. Telemed. Telecare 2019, 25, 378-385. [CrossRef]

20. Choi, W.S.; Choi, J.H.; Oh, J.; Shin, I.S.; Yang, J.S. Effects of Remote Monitoring of Blood Pressure in Management of Urban Hypertensive Patients: A Systematic Review and Meta-Analysis. Telemed. J. e-Health 2020, 26, 744-759. [CrossRef]

21. Jovell, A.J.; Navarro-Rubio, M.D. [Evaluation of scientific evidence]. Med. Clin. 1995, 105, 740-743.

22. Wosik, J.; Fudim, M.; Cameron, B.; Gellad, Z.F.; Cho, A.; Phinney, D.; Curtis, S.; Roman, M.; Poon, E.G.; Ferranti, J.; et al. Telehealth transformation: COVID-19 and the rise of virtual care. J. Am. Med. Inform. Assoc. 2020, 27, 957-962. [CrossRef]

23. Cowie, M.R.; Lam, C.S.P. Remote monitoring and digital health tools in CVD management. Nat. Rev. Cardiol. 2021, 18, 457-458. [CrossRef]

24. Higgins, J.P.T.; Green, S. Cochrane Handbook for Systematic Reviews of Interventions; Version 502 [updated September 2009]; Wiley: New York, NY, USA, 2009.

25. Bosworth, H.B.; Olsen, M.K.; McCant, F.; Harrelson, M.; Gentry, P.; Rose, C.; Goldstein, M.K.; Hoffman, B.B.; Powers, B.; Oddone, E.Z. Hypertension Intervention Nurse Telemedicine Study (HINTS): Testing a multifactorial tailored behavioral/educational and a medication management intervention for blood pressure control. J. Am. Heart Assoc. 2007, 153, 918-924. [CrossRef]

26. Kerry, S.; Markus, H.; Khong, T.; Cloud, G.; Tulloch, J.; Coster, D.; Ibison, J.; Oakeshott, P. Home blood pressure monitoring with nurse-led telephone support among patients with hypertension and a history of stroke: A community-based randomized controlled trial. CMAJ 2012, 185, 23-31. [CrossRef]

27. Pan, F.; Wu, H.; Liu, C.; Zhang, X.; Peng, W.; Wei, X.; Gao, W. Effects of home telemonitoring on the control of high blood pressure: A randomised control trial in the Fangzhuang Community Health Center, Beijing. Aust. J. Prim. Health 2018, 24, 398-403. [CrossRef]

28. Zha, P.; Qureshi, R.; Porter, S.; Chao, Y.Y.; Pacquiao, D.; Chase, S.; O’Brien-Richardson, P. Utilizing a Mobile Health Intervention to Manage Hypertension in an Underserved Community. West. J. Nurs. Res. 2020, 42, 201-209. [CrossRef]

29. Artinian, N.T.; Flack, J.M.; Nordstrom, C.K.; Hockman, E.M.; Washington, O.G.; Jen, K.L.; Fathy, M. Effects of nurse-managed telemonitoring on blood pressure at 12-month follow-up among urban African Americans. Nurs. Res. 2007, 56, 312-322. [CrossRef] [PubMed]

30. Cicolini, G.; Simonetti, V.; Comparcini, D.; Celiberti, I.; Di Nicola, M.; Capasso, L.M.; Flacco, M.E.; Bucci, M.; Mezzetti, A.; Manzoli, L. Efficacy of a nurse-led email reminder program for cardiovascular prevention risk reduction in hypertensive patients: A randomized controlled trial. Int. J. Nurs. Stud. 2014, 51, 833-843. [CrossRef] [PubMed]

31. Hebert, P.; Sisk, J.; Tuzzio, L.; Casabianca, J.; Pogue, V.; Wang, J.; Chen, Y.; Cowles, C.; McLaughlin, M.A. Nurse-led disease management for hypertension control in a diverse urban community: A randomized trial. J. Gen. Intern. Med. 2012, 27, 630-639. [CrossRef] [PubMed]

32. Kim, K.B.; Han, H.R.; Huh, B.; Nguyen, T.; Lee, H.; Kim, M.T. The effect of a community-based self-help multimodal behavioral intervention in Korean American seniors with high blood pressure. Am. J. Hypertens. 2014, 27, 1199-1208. [CrossRef] [PubMed]

33. Magda, M.M.; Riad, N.A.; Badawy, A.E.; EI Gafar, S.E.A.; El-Hammed, B.M.A.; Eltomy, E.M. Tele-nursing versus Routine Outpatient Teaching for Improving Arterial Blood Pressure and Body Mass Index for Hypertensive Patients. Am. J. Nurs. Res. 2020, 8, 18-26.

34. Pour, E.R.; Aliyari, S.; Farsi, Z.; Ghelich, Y. Comparing the effects of interactive and noninteractive education using short message service on treatment adherence and blood pressure among patients with hypertension. Nurs. Midwifery Stud. 2020, 9, 68-76.

35. Rubinstein, A.; Miranda, J.J.; Beratarrechea, A.; Diez-Canseco, F.; Kanter, R.; Gutierrez, L.; Bernabé-Ortiz, A.; Irazola, V.; Fernandez, A.; Letona, P.; et al. Effectiveness of an mHealth intervention to improve the cardiometabolic profile of people with prehypertension in low-resource urban settings in Latin America: A randomised controlled trial. Lancet Diabetes Endocrinol. 2016, 2016 4, 52-63. [CrossRef]

36. The Cochrane Collaboration. Review Manager (RevMan)[Computer Program], Version 5.4 for Windows; The Cochrane Collaboration: Oxford, UK, 2020.

37. Egger, M.; Davey-Smith, G.; Altman, D. (Eds.) Systematic Reviews in Healthcare: Meta-Analysis in Context; John Wiley \& Sons: New York, NY, USA, 2008.

38. Takeshima, N.; Sozu, T.; Tajika, A.; Ogawa, Y.; Hayasaka, Y.; Furukawa, T.A. Which is more generalizable, powerful and interpretable in meta-analyses, mean difference or standardized mean difference? BMC Med. Res. Methodol. 2014, 14, 30. [CrossRef]

39. Cohen, J. Statistical Power Analysis for the Behavioral Sciences; Lawrence Erlbaum Associates: New York, NY, USA, 1988.

40. Higgins, J.P.; Thompson, S.G.; Deeks, J.J.; Altman, D.G. Measuring inconsistency in meta-analyses. BMJ 2003, 327, 557-560. [CrossRef]

41. Hill, M.; Bone, L.; Hilton, S.; Roary, M.; Kelen, G.; Levine, D. A clinical trial to improve high blood pressure care in young urban black men: Recruitment, follow-up, and outcomes. Am. J. Hypertens. 1999, 12, 548-554. [CrossRef]

42. Friedman, R.H.; Kazis, L.E.; Jette, A.; Smith, M.B.; Stollerman, J.; Torgerson, J.; Carey, K. A telecommunications system for monitoring and counseling patients with hypertension: Impact on medication adherence and blood pressure control. Am. J. Hypertens. 1996, 9, 285-292. [CrossRef] 
43. McMahon, G.; Gomes, H.; Hickson, H.S.; Hu, T.; Levine, B.; Conlin, P. Web-based care management in patients with poorly controlled diabetes. Diabetes Care 2005, 28, 1624-1629. [CrossRef]

44. Shea, S.; Weinstock, R.; Starren, J.; Teresi, J.; Palmas, W.; Field, L.; Morin, P.; Goland, R.; Izquierdo, R.E.; Wolff, L.T.; et al. A randomized trial comparing telemedicine case management with usual care in older, ethnically diverse, medically underserved patients with diabetes mellitus. J. Am. Med. Inform. Assoc. 2006, 13, 40-51. [CrossRef] [PubMed]

45. Carrasco, M.P.; Salvador, C.H.; Sagredo, P.G.; Marquez-Montes, J.; de Mingo, M.A.G.; Fragua, J.A.; Rodríguez, M.C.; García-Olmos, L.M.; García-López, F.; Muñoz Carrero, A.; et al. Impact of patient-general practitioner short-messages-based interaction on the control of hypertension in a follow-up service for low-to-medium risk hypertensive patients: A randomized controlled trial. IEEE Trans. Inf. Technol. Biomed. 2008, 12, 780-791. [CrossRef]

46. Green, B.B.; Cook, A.J.; Ralston, J.D.; Fishman, P.A.; Catz, S.L.; Carlson, J. Effectiveness of home blood pressure monitoring, Web communication, and pharmacist care on hypertension control: A randomized controlled trial. JAMA 2008, 299, 2857-2867. [CrossRef]

47. Madsen, L.B.; Kirkegaard, P.; Pedersen, E.B. Blood pressure control during telemonitoring of home blood pressure. A randomized controlled trial during 6 months. Blood Press. 2008, 17, 78-86. [CrossRef] [PubMed]

48. Parati, G.; Omboni, S.; Albini, F.; Piantoni, L.; Giuliano, A.; Revera, M.; Illyes, M.; Mancia, G.; Tele, B.S.G. Home blood pressure telemonitoring improves hypertension control in general practice. The TeleBPCare study. J. Hypertens. 2009, 27, 198-203. [CrossRef] [PubMed]

49. Park, M.; Kim, H.; Kim, K. Cellular phone and Internet-based individual intervention on blood pressure and obesity in obese patients with hypertension. Int. J. Med. Inform. 2009, 78, 704-710. [CrossRef] [PubMed]

50. Varis, J.; Kantola, I. The choice of home blood pressure result reporting method is essential: Results mailed to physicians did not improve hypertension control compared with ordinary office-based blood pressure treatment. Blood Press. 2010, 19, 319-324. [CrossRef]

51. Hoffmann-Petersen, N.; Lauritzen, T.; Bech, J.N.; Pedersen, E.B. Short-term telemedical home blood pressure monitoring does not improve blood pressure in uncomplicated hypertensive patients. J. Hum. Hypertens. 2017, 31, 93-98. [CrossRef] [PubMed]

52. Ionov, M.V.; Zhukova, O.V.; Yudina, Y.S.; Avdonina, N.G.; Emelyanov, I.V.; Kurapeev, D.I.; Zvartau, N.E.; Konradi, A.O. Valuebased approach to blood pressure telemonitoring and remote counseling in hypertensive patients. Blood Press. 2020, 30, 1-11. [CrossRef]

53. Logan, A.G.; Irvine, M.J.; McIsaac, W.J.; Tisler, A.; Rossos, P.G.; Easty, A.; Feig, D.S.; Cafazzo, J.A. Effect of Home Blood Pressure Telemonitoring With Self-Care Support on Uncontrolled Systolic Hypertension in Diabetics. Hypertension 2012, 60, 51-57. [CrossRef]

54. Neumann, C.L.; Menne, J.; Rieken, E.M.; Fischer, N.; Weber, M.H.; Haller, H.; Schulz, E.G. Blood pressure telemonitoring is useful to achieve blood pressure control in inadequately treated patients with arterial hypertension. J. Hum. Hypertens. 2011, 25, 732-738. [CrossRef] [PubMed]

55. Wakefield, B.J.; Holman, J.E.; Ray, A.; Scherubel, M.; Adams, M.R.; Hillis, S.L.; Rosenthal, G.E. Effectiveness of home telehealth in comorbid diabetes and hypertension: A randomized, controlled trial. Telemed. J. e-Health 2011, 17, 254-261. [CrossRef]

56. Bosworth, H.B.; Powersm, B.J.; Olsen, M.K.; McCant, F.; Grubber, J.; Smith, V.; Gentry, P.W.; Rose, C.; Van Houtven, C.; Wang, V.; et al. Home blood pressure management and improved blood pressure control: Results from a randomized controlled trial. Arch. Intern. Med. 2011, 171, 1173-1180. [CrossRef]

57. Migneault, J.; Dedier, J.; Wright, J.; Heeren, T.; Campbell, M.; Morisky, D.; Rudd, P.; Friedman, R.H. A culturally adapted telecommunication system to improve physical activity, diet quality, and medication adherence among hypertensive AfricanAmericans: A randomized controlled trial. Ann. Behav. Med. 2012, 43, 62-73. [CrossRef] [PubMed]

58. Park, M.; Kim, H. Evaluation of mobile phone and Internet intervention on waist circumference and blood pressure in postmenopausal women with abdominal obesity. Int. J. Med. Eng. Inform. 2012, 81, 388-394. [CrossRef] [PubMed]

59. Bove, A.; Homko, C.; Santamore, W.; Kashem, M.; Kerper, M.; Elliott, D. Managing hypertension in urban underserved subjects using telemedicine-a clinical trial. Am. Heart J. 2013, 165, 615-621. [CrossRef]

60. Wakefield, B.J.; Koopman, R.J.; Keplinger, L.E.; Bomar, M.; Bernt, B.; Johanning, J.L.; Kruse, R.L.; Davis, J.W.; Wakefield, D.S.; Mehr, D.R. Effect of home telemonitoring on glycemic and blood pressure control in primary care clinic patients with diabetes. Telemed. J. e-Health 2014, 20, 199-205. [CrossRef]

61. Yi, S.; Tabaei, B.; Angell, S.; Rapin, A.; Buck, M.; Pagano, W.; Maselli, F.J.; Simmons, A. Self-blood pressure monitoring in an urban, ethnically diverse population: A randomized clinical trial utilizing the electronic health record. Circ. Cardiovasc. Qual. Outcomes 2015, 8, 138-145. [CrossRef]

62. Higgins, J.P.T.; Savović, J.; Page, M.J.; Elbers, R.G.; Sterne, J.A.C. Chapter 8: Assessing risk of bias in a randomized trial. In Cochrane Handbook for Systematic Reviews of Interventions; Higgins, J., Thomas, J., Eds.; Cochrane: London, UK, 2021; Available online: wwwtrainingcochraneorg/handbook (accessed on 5 August 2021).

63. Duval, S.; Tweedie, R. Trim and fill: A simple funnel-plot-based method of testing and adjusting for publication bias in meta-analysis. Biometrics 2000, 56(2), 455-463. [CrossRef]

64. Sterne, J.A.; Egger, M. Funnel plots for detecting bias in meta-analysis: Guidelines on choice of axis. J. Clin. Epidemiol. 2001, 54, 1046-1055. [CrossRef]

65. OECD Indicators. Health at a Glance 2019: OECD Indicators; OECD Publishing: Paris, French, 2019. 
66. Hwang, J.Y.; Kim, K.Y.; Lee, K.H. Factors that influence the acceptance of telemetry by emergency medical technicians in ambulances: An application of the extended technology acceptance model. Telemed. J. e-Health 2014, 20, 1127-1134. [CrossRef]

67. National Center for Health Statistics (US). Health, United States, 2013: With Special Feature on Prescription Drugs. National Center for Health Statistics (US): Hyattsville, MD, USA, 2014.

68. Neal, B.; MacMahon, S.; Chapman, N. Effects of ACE inhibitors, calcium antagonists, and other blood-pressure-lowering drugs: Results of prospectively designed overviews of randomised trials. Blood Pressure Lowering Treatment Trialists' Collaboration. Lancet 2000, 356, 1955-1964. [PubMed]

69. Cook, N.R.; Cohen, J.; Hebert, P.R.; Taylor, J.O. Hennekens, C.H. Implications of small reductions in diastolic blood pressure for primary prevention. Arch Intern. Med. 1995, 155, 701-709. [CrossRef]

70. Law, M.R.; Morris, J.K.; Wald, N.J. Use of blood pressure lowering drugs in the prevention of cardiovascular disease: Metaanalysis of 147 randomised trials in the context of expectations from prospective epidemiological studies. BMJ 2009, 338, b1665. [CrossRef]

71. Hammersley, V.; Parker, R.; Paterson, M.; Hanley, J.; Pinnock, H.; Padfield, P.; Stoddart, A.; Park, H.G.; Sheikh, A.; McKinstry, B. Telemonitoring at scale for hypertension in primary care: An implementation study. PLoS Med. 2020, 17, e1003124. [CrossRef]

72. Omboni, S. Telemedicine During the COVID-19 in Italy: A Missed Opportunity? Telemed. J. e-Health 2020, 26, 973-975. [CrossRef]

73. Margolis, K.L.; Asche, S.E.; Dehmer, S.P.; Bergdall, A.R.; Green., B.B.; Sperl-Hillen, J.M.; Nyboer, R.A.; Pawloski, P.A.; Maciosek, M.V.; Trower, N.K.; et al. Long-term Outcomes of the Effects of Home Blood Pressure Telemonitoring and Pharmacist Management on Blood Pressure Among Adults With Uncontrolled Hypertension: Follow-up of a Cluster Randomized Clinical Trial. JAMA Netw. Open 2018, 1, e181617. [CrossRef] [PubMed]

74. Williams, B.; Mancia, G.; Spiering, W.; Rosei, E.A.; Azizi, M.; Burnier, M.; Clement, D.; Coca, A.; De Simone, G.; Dominiczak, A.; et al. 2018 Practice Guidelines for the management of arterial hypertension of the European Society of Hypertension and the European Society of Cardiology: ESH/ESC Task Force for the Management of Arterial Hypertension. J. Hypertens. 2018, 36, 2284-2309. [CrossRef] [PubMed]

75. Hjelm, N.M. Benefits and drawbacks of telemedicine. J. Telemed. Telecare 2005, 11, 60-70. [CrossRef] [PubMed]

76. Filchev, R.; Pavlova, D.; Dimova, R.; Dovramadjiev, T. Healthcare System Sustainability by Application of Advanced Technologies in Telemedicine and eHealth. In International Conference on Human Interaction and Emerging Technologies; Springer: Cham, Switzerland, 2021; pp. 1011-1017.

77. Chu, D.K.; Akl, E.A.; Duda, S.; Solo, K.; Yaacoub, S.; Schünemann, H.J. Physical distancing, face masks, and eye protection to prevent person-to-person transmission of SARS-CoV-2 and COVID-19: A systematic review and meta-analysis. Lancet 2020, 395, 1973-1987. [CrossRef] 